\title{
The Quasi-Classical Limit of Quantum Scattering Theory
}

\author{
Kenji Yajima \\ Mathematical Research Institute, ETH-Zürich, Switzerland, and Department of \\ Mathematics, University of Tokyo, Tokyo, Japan ${ }^{* *}$
}

\begin{abstract}
We study the quasi-classical limit of the quantum mechanical scattering operator for non-relativistic simple scattering system. The connection between the quantum mechanical and classical mechanical scattering theories is obtained by considering the asymptotic behavior as $\hbar \rightarrow 0$ of the quantum mechanical scattering operator on the state $\exp (-i p \cdot a / \hbar) f(p)$ in the momentum representation.
\end{abstract}

\section{Introduction}

Let us consider the Schrödinger operator

$$
H^{h}=-\frac{\hbar^{2}}{2 m} \Delta+V(x), \quad \Delta=\frac{\partial^{2}}{\partial x_{1}^{2}}+\ldots+\frac{\partial^{2}}{\partial x_{1}^{2}}
$$

in the Hilbert space $\mathscr{H}=L^{2}\left(\mathbb{R}^{n}\right)$ and let $H_{0}^{h}=-\frac{\hbar^{2}}{2 m} \Delta$. Here $\hbar=\frac{h}{2 \pi}$ and $h$ is the small positive parameter called Planck's constant. We assume the potential $V(x)$ to satisfy the following condition.

Assumption (A). (1) $V(x)$ is a real valued infinitely differentiable function on $\mathbb{R}^{n}$.

(2) For any multi-index $\alpha$, there exist constants $m(\alpha)>|\alpha|+1$ and $C_{\alpha}>0$ such that

$$
\left|\left(\frac{\partial}{\partial x}\right)^{\alpha} V(x)\right| \leqq C_{\alpha}(1+|x|)^{-m(\alpha)} .
$$

Under this condition $H_{0}^{h}$ and $H^{h}$ are self-adjoint operators with the domain $\mathscr{D}\left(H_{0}^{h}\right)=\mathscr{D}\left(H^{h}\right)=H^{2}\left(\mathbb{R}^{n}\right)=$ the Sobolev space of order 2. Furthermore it is well known that the wave operators $W_{ \pm}^{h}$ defined as

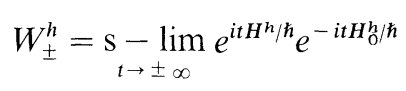

exist and are complete:

* Partially supported by Fûjû-kai Foundation and Sakkô-kai Foundation

** Current address 


$$
\begin{gathered}
\mathscr{R}\left(W_{+}^{h}\right)=\mathscr{R}\left(W_{-}^{h}\right)=\text { the spectrally absolutely continuous subspace of } \mathscr{H} \\
\text { w.r.t. } H^{h} \text {. }
\end{gathered}
$$

(See Agmon [1] and Kuroda [10] for more general results.) The scattering operator $S^{h}$ for the system is defined as

$$
S^{h}=\left(W_{+}^{h}\right)^{*} W_{-}^{h}
$$

and is therefore a unitary operator on $\mathscr{H}$. We shall study the asymptotic behavior of the operator $S^{h}$ as $h \rightarrow 0$ and its relation to the corresponding classical mechanical scattering theory for the Hamiltonian

$$
H(x, \xi)=\xi^{2} / 2 m+V(x) .
$$

In the classical mechanical scattering theory, the following results are wellknown under the Assumption (A): i) For any $(a, \eta) \in \mathbb{R}^{n} \times \mathbb{R}^{n}(\eta \neq 0)$ there exists a unique solution $\left(x_{-}(t, a, \eta), \xi_{-}(t, a, \eta)\right)$ of the equation of motion

$$
\frac{d x}{d t}=\frac{\xi}{m}, \frac{d \xi}{d t}=-\operatorname{grad} V(x)
$$

such that

$$
\begin{aligned}
& \lim _{t \rightarrow-\infty}\left|x_{-}(t, a, \eta)-t \eta / m-a\right|=0, \\
& \lim _{t \rightarrow-\infty}\left|\xi_{-}(t, a, \eta)-\eta\right|=0 ;
\end{aligned}
$$

ii) there exists a closed null set (i.e., Lebesgue measure zero) $e \subset \mathbb{R}^{n} \times \mathbb{R}^{n}$ such that for any $(a, \eta) \notin e$, there exists $\left(a_{+}(a, \eta), \eta_{+}(a, \eta)\right) \in \mathbb{R}^{n} \times \mathbb{R}^{n}$ such that

$$
\begin{aligned}
& \lim _{t \rightarrow \infty}\left|x_{-}(t, a, \eta)-t \eta_{+}(a, \eta) / m-a_{+}(a, \eta)\right|=0, \\
& \lim _{t \rightarrow \infty}\left|\xi_{-}(t, a, \eta)-\eta_{+}(a, \eta)\right|=0
\end{aligned}
$$

iii) the classical scattering operator $S^{c l}$ defined on $\mathbb{R}^{n} \times \mathbb{R}^{n} \backslash e$ as $S^{c l}(a, \eta)=$ $\left(a_{+}(a, \eta), \eta_{+}(a, \eta)\right)$ is a $C^{1}$-canonical mapping.

We define as $e(a)=\left\{\eta \in \mathbb{R}^{n} ;(a, \eta) \in e\right\} \cup\{0\}$. Obviously $e(a)$ is a null set for almost all $a \in \mathbb{R}^{n}$, i.e., $e(a)$ has Lebesgue measure zero in $\mathbb{R}^{n}$. (See Herbst [6], Simon [12] and Huniziker [9]). We write as $\dot{x}(t)=\frac{d x}{d t}$ and $\dot{\xi}(t)=\frac{d \xi}{d t}$.

It will be proved that for any $a \in \mathbb{R}^{n}, \eta_{+}(a, \eta)$ and $a_{+}(a, \eta)$ are smooth functions of $\eta \in \mathbb{R}^{n} \backslash e(a)$. We define as

$$
e(a)^{e x}=\left\{\eta \in \mathbb{R}^{n} \backslash e(a): \operatorname{det}\left[\partial \eta_{+}(a, \eta) / \partial \eta\right]=0\right\} \cup e(a) .
$$

For any $f \in \mathscr{H}$, let us write as $f_{a}^{h}(\eta)=e^{-i(a \cdot \eta / \hbar)} f(\eta)$. Our main theorem can be stated as follows.

Theorem. Let Assumption (A) be satisfied. Let $a \in \mathbb{R}^{n}$ and let $e(a)^{e x}$ be defined as (0.6). Then for any $f \in \mathscr{H}$ with supp $f$ a compact subset of $\mathbb{R}^{n} \backslash e(a)^{e x}$ 


$$
\begin{aligned}
& \lim _{h \downarrow 0} \|\left(\hat{S}^{h} f_{a}^{h}\right)(\eta)- \sum_{\eta=\eta+\left(a, \eta_{J}\right)} e^{i \operatorname{lnd} \gamma\left(\eta, \eta_{j}\right) \pi / 2+i\left(S\left(\eta, \eta_{J}\right)-\eta \cdot a_{+}\left(a, \eta_{J}\right)\right) / \hbar} \\
&\left.\cdot\left|\operatorname{det}\left[\partial \eta_{+}(a, \eta) / \partial \eta\right]\right|_{\eta=\eta_{J}}\right|^{-1 / 2} f\left(\eta_{j}(\eta)\right) \|=0
\end{aligned}
$$

where the summation is taken over all $\eta_{j}$ 's satisfying $\eta=\eta_{+}\left(a, \eta_{j}\right)$.

Here $\hat{S}^{h}$ is the scattering operator in momentum representation: $\hat{S}^{h}=\mathscr{F}^{h} S^{h}\left(\mathscr{F}^{h}\right)^{-1}$,

$$
\mathscr{F}^{h} f(\eta)=h^{-n / 2} \int e^{-i x \cdot \eta / \hbar} f(x) d x ;
$$

Ind $\gamma\left(\eta, \eta_{j}\right)$ is so-called Keller-Maslov-index of the orbit $\left\{x_{-}\left(t, a, \eta_{j}\right), \xi_{-}\left(t, a, \eta_{j}\right)\right\}$; $S\left(\eta, \eta_{j}\right)$ is defined as

$$
S\left(\eta, \eta_{j}\right)=\lim _{\substack{t \rightarrow \infty \\ s \rightarrow-\infty}}\left\{\int_{s}^{t} L\left(x\left(u, a, \eta_{j}\right), \dot{x}\left(u, a, \eta_{j}\right)\right) d u+\frac{s}{2 m} \eta_{j}^{2}-\frac{t}{2 m} \eta^{2}\right\},
$$

where $L(x, \dot{x})=\frac{m}{2} \dot{x}^{2}-V(x)$ is the Lagrangian of the system.

The connection between the quantum mechanics $(0.1)$ and the classical mechanics $(0.2)$ has been discussed since the advent of the quantum mechanics and there are several mathematically rigorous arguments. The refined form of WKBJmethod by Maslov [11] and the rigorous form of Ehrenfest's theorem by Hepp [4] seem to be outstanding, among others. However, unfortunately, these works are mostly concerned with the dynamics in finite time and the connection of the scattering theories has not been discussed (see, however, Hepp's unpublished note [5]). In this paper we study the connection of these scattering theories using the WKBJ-method which seems to be fit best for the purpose. The main tools for proving the theorem are the approximate fundamental solution constructed by Fujiwara [3] and the $L^{2}$-boundedness theorem for certain kind of oscillatory integral operators by Asada-Fujiwara [2].

Before proceeding to the text we want to discuss here about a meaning of the theorem. For a smooth real function $S(\eta)$, we can consider the wave function $e^{-i S(\eta) / \hbar} f(\eta)$ to represent, asymptotically as $\hbar \rightarrow 0$, the ensemble of classical particles $\left(\frac{\partial S}{\partial \eta}(\eta), \eta\right)$ with momentum distribution $|f(\eta)|^{2} d \eta$. Hence by taking $e^{-i a \cdot \eta / \hbar} f(\eta)$ as an initial state, we prepare it as to represent the ensemble of particles concentrated at the configuration $x=a$ with momentum distribution $|f(\eta)|^{2} d \eta$. Thus the incoming particles are prepared as $x(t) \sim a+t \eta / m, \xi(t) \sim \eta$ at remote past. What the theorem says is that the final state $\hat{S}^{h}\left(e^{-i a \cdot \eta / \hbar} f\right)$ is asymptotically represented as the incoherent superposition of the wave functions

$$
e^{i \operatorname{Ind} \gamma\left(\eta, \eta_{J}\right) \pi / 2+i\left(S\left(\eta, \eta_{J}\right)-\eta \cdot a+\left(a, \eta_{J}\right)\right) / \hbar}\left|\operatorname{det}\left(\frac{\partial \eta_{+}}{\partial \eta}\left(a, \eta_{j}\right)\right)\right|^{-1 / 2} f\left(\eta_{j}\right)
$$

which represents the particles

$$
\left(-\frac{\partial}{\partial \eta}\left(S\left(\eta, \eta_{j}\right)-\eta \cdot a_{+}\left(a, \eta_{j}\right)\right), \eta\right)=\left(a_{+}\left(a, \eta_{j}\right), \eta_{+}\left(a, \eta_{j}\right)\right)
$$


with the momentum distribution

$$
\left|\operatorname{det}\left(\frac{\partial \eta_{+}}{\partial \eta_{j}}\right)\left(a, \eta_{j}\right)\right|^{-1}\left|f\left(\eta_{j}\right)\right|^{2} d \eta=\left|f\left(\eta_{j}\right)\right|^{2} d \eta_{j}
$$

Moreover, since $a_{+}\left(a, \eta_{k}\right) \neq a_{+}\left(a, \eta_{j}\right)$ for $j \neq k$, by the canonical property of $S^{c l}$, this incoherent superposition turns to the coherent one, as $h \rightarrow 0$. Thus we may say that the classical mechanical scattering can be represented as a limit of $h \rightarrow 0$ of the quantum scattering theory.

The plan of the paper is as follows. In Sect. 1 we shall study the asymptotic behavior as $h \rightarrow 0$ of the wave packet

$$
e^{-i t H_{0}^{\epsilon} / h} \tilde{f}_{a}^{h}, \tilde{f}_{a}^{\hbar h}(x)=h^{-n / 2} \int e^{i x \cdot \eta / h} f_{a}^{h}(\eta) d \eta
$$

In Sects. 2 and 3, we shall prepare the materials from the classical mechanics which are necessary in the following sections. In Sect. 4 we shall study the asymptotic behavior of $W_{-}^{h} \tilde{f}_{a}^{h}$. In Sects. 5 and 6 , we shall study $\hat{S}^{h} f_{a}^{h}$. In the last section, 7, some remarks will be given. Especially a result which is related to Dollard's cone scattering theory [13] will be obtained.

We list here the notation and the conventions used in the paper.

For domains $\Omega_{1}$ and $\Omega_{2}, \Omega_{1} \subset \subset \Omega_{2}$ means that $\Omega_{1}$ is a precompact subset of $\Omega_{2}$ and $\bar{\Omega}_{1}$, the closure of $\Omega_{1}$, is contained in $\Omega_{2} . L^{2}(\Omega)$ is the Hilbert space of all square integrable functions on $\Omega$ equipped with the inner product and the norm as

$$
(f, g)=\int_{\Omega} f(x) \cdot \overline{g(x)} d x,\|f\|=(f, f)^{1 / 2} .
$$

If $\Omega=\mathbb{R}^{n}$, we simply write as $L^{2}\left(\mathbb{R}^{n}\right)=\mathscr{H}$. For $x=\left(x_{1}, \ldots, x_{n}\right) \in \mathbb{R}^{n},|x|=\left(\sum x_{j}^{2}\right)^{1 / 2}$ is the Euclidean norm. For the multi-index $\left(\alpha_{1}, \ldots, \alpha_{n}\right)=\alpha, \alpha_{j} \in \mathbb{N},|\alpha|=\sum \alpha_{j}$. $x^{\alpha}=x_{1}^{\alpha_{1}} \ldots x_{n}^{\alpha_{n}}$ and $(\partial / \partial x)^{\alpha}=\left(\partial / \partial x_{1}\right)^{\alpha_{1}} \ldots\left(\partial / \partial x_{n}\right)^{\alpha_{n}} \cdot d x=d x_{1} \ldots d x_{n} . \mathscr{B}\left(\mathbb{R}^{n}\right)$ is the space of all $C^{\infty}$-bounded functions with their derivatives. For vector-valued function $f(x), \frac{\partial f}{\partial x}$ stands for the matrix $\left(\frac{\partial f_{j}}{\partial x_{k}}\right)_{j, k}$. For matrix $A=\left(a_{i j}\right),|A|=$ $\sup _{i}\left\{\sum_{j} a_{i j}^{2}\right\}^{1 / 2}$. For $h>0, \mathscr{F}^{h}$ is the Fourier transform defined by $(0.8)$ and if $\hbar=1$, we omit the index $h$. For the Fourier transform we write as $\hat{f}^{h}=\mathscr{F}^{h} f$, $\tilde{f}^{h}=\mathscr{F}^{h} * f=\left(\mathscr{F}^{h}\right)^{-1} f \cdot f_{a}^{h}(\eta)=e^{-i \eta \cdot a / h} f(\eta)$ and $\tilde{f}_{a}^{h}=\mathscr{F}^{h} * f_{a}^{h}$.

$\pi_{1}$ and $\pi_{2}$ are the projections from $\mathbb{R}^{n} \times \mathbb{R}^{n}$ to $\mathbb{R}^{n}: \pi_{1}(x, p)=x$ and $\pi_{2}(x, p)=p$.

For $a \in \mathbb{R}^{n}$ we define as $\pi_{2, a}^{*}(p)=(a, p)$ and $\pi_{1}^{*}(x)=(x, 0)$. For an operator $T$ in a Hilbert space $\mathscr{X}, \mathscr{D}(T), \mathscr{R}(T)$ stand for the domain and the range of the operator. The constants appearing in the formulas are distinguished in one context but in the other they are not distinguished and may be written by the same symbol if it is clear that which constants are meant. We sometimes omit the indices and suffices or other parameters if no confusions are feared. 


\section{Uniform Estimate for the Free Propagator}

In Sect. 4 we shall study the asymptotic behavior of the wave operator $W_{-}^{h}$ on the coherent state $\tilde{f}_{a}^{h}$. As a starting point we study here the asymptotic behavior of $\exp \left(-i t H_{0}^{h} / \hbar\right) \tilde{f}_{a}^{h}$ as $h \rightarrow 0$. Actually we prove the following theorem, the essential feature of which is that the estimate is uniform with respect to time $t$ outside $t=0$.

Theorem 1.1. For any $a \in \mathbb{R}^{n}$ and $t>0$ let $u$ s define the operator $T_{0, a}^{h}(t)$ on $\mathscr{H}$ as

$$
T_{0, a}^{h}(t) f(x)=e^{i m(x-a)^{2} / 2 t h}(m / i t)^{n / 2} f(m(x-a) / t) .
$$

Then

1) $T_{0, a}^{h}(t)$ is a unitary operator in $\mathscr{H}$ and is strongly continuous in $t>0$;

2) for any $\delta>0$,

$$
\lim _{h \rightarrow 0} \sup _{|t| \geqq \delta}\left\|e^{-i t H_{0}^{h / \hbar} \tilde{f}_{a}^{h}}-T_{0, a}^{h}(t) f\right\|=0 .
$$

Proof. Since $\exp \left(-i t H_{0}^{h} / \hbar\right) \tilde{f}_{a}^{h}(x)=\exp \left(i t H_{0}^{h} / \hbar\right) \tilde{f}_{0}^{h}(x-a) \quad$ and $\quad T_{0, a}^{h}(t) f(x)=$ $T_{0,0}^{h}(t) f(x-a)$, it is sufficient to prove 1$)$ and 2$)$ for the case $a=0$. In what follows we omit the index $a=0$. Since the first statement is clear, we prove 2 ) only. Since $T_{0}^{h}(t)$ and $\exp \left(-i t H_{0}^{h} / \hbar\right) \mathscr{F}^{h *}$ are unitary, the standard limiting procedure shows that it suffices to prove (1.2) for $f \in C_{0}^{\infty}\left(\mathbb{R}^{n}\right)$. By the Plancherel's theorem

$$
\begin{aligned}
e^{-t t H_{0}^{h} / \hbar} \tilde{f}^{h}(x)= & h^{-n / 2} \int e^{\left(x \cdot p-p^{2} t / 2 m\right) / \hbar} f(p) d p \\
= & h^{-n / 2} e^{i m x^{2} / 2 t \hbar} \int e^{-i t(p-m x / t)^{2} / 2 m \hbar} f(p) d p \\
= & h^{-n / 2} e^{i m x^{2} / 2 t h}(m \hbar / i t)^{n / 2} \int e^{i m h y^{2} / 2 t-i m x \cdot y / t} \hat{f}(y) d y \\
= & T_{0}^{h}(t) f+e^{i m x^{2} / 2 t \hbar}(m / i t)^{n / 2}(2 \pi)^{-n / 2} \\
& \cdot \int_{0}^{1} \int_{\mathbb{R}^{n}}(i m \hbar / 2 t) e^{-i m x \cdot y / t} e^{i m \hbar s y^{2} / 2 t} y^{2} \hat{f}(y) d y d s .
\end{aligned}
$$

Hence by Minkowski's inequality and the Parseval relation we get

$$
\left\|e^{-i t H_{0} / \hbar} \hat{f}^{h}-T_{0}^{h}(t) f\right\| \leqq(m \hbar / 2 t)\left\|y^{2} \hat{f}(y)\right\| \leqq(m \hbar / 2 t)\|f\|_{2},
$$

which implies the relation (1.2).

(Q.E.D.)

\section{Properties of Classical Orbit}

In this section we study several properties of the motion of classical particle which will be needed in the following sections. We write the canonical transformations in the phase space $\mathbb{R}^{n} \times \mathbb{R}^{n}$ associated with the Hamiltonians $H(x, \xi)=$ $\xi^{2} / 2 m+V(x)$ and $H_{0}(\xi)=\xi^{2} / 2 m$ as $U(t)$ and $U_{0}(t)$, that is,

$$
U_{0}(t)\left(x_{0}, \xi_{0}\right)=\left(x_{0}+t \xi_{0} / m, \xi_{0}\right)
$$

and

$$
U(t)\left(x_{0}, \xi_{0}\right)=\left(x_{t}, \xi_{t}\right),
$$


where $\left(x_{t}, \xi_{t}\right)$ is the solution of the Hamilton equation

$$
\begin{aligned}
& \dot{x}(t)=\xi(t) / m, \quad \xi(t)=-\operatorname{grad}_{x} V(x(t)), \\
& x(0)=x_{0}, \quad \xi(0)=\xi_{0} .
\end{aligned}
$$

In the followings we always assume Assumption (A). For $(a, \eta) \in \mathbb{R}^{n} \times \mathbb{R}^{n}$ we write as $U(t) U_{0}(s)(a, \eta)=\left(x_{s}(t, a, \eta), \xi_{s}(t, a, \eta)\right)$.

Lemma 2.1. Let $K \subset \subset \mathbb{R}^{n} \backslash\{0\}$. Then there exists a constant $R>0$ depending only on $K$ such that for $s<0$ with sufficiently large $|s|$ and any multi-index $\alpha$, there exists a constant $C_{\alpha}$ such that for any $\eta \in K$ and $0 \leqq t \leqq|s+R|$

$$
\begin{aligned}
& \mid\left(\frac{\partial}{\partial \eta}\right)^{\alpha} x_{s}(t, a, \eta)-(s+t) m^{-1} \frac{\partial^{\alpha} \eta}{\partial \eta^{\alpha}}-\frac{\partial^{\alpha} a}{\partial \eta^{\alpha}} \mid \\
& \leqq(2 m)^{-1}|s+t|\left|\frac{\partial^{\alpha} \eta}{\partial \eta^{\alpha}}\right|+C_{\alpha}|s+t|^{-\varepsilon}, \\
&\left|\left(\frac{\partial}{\partial \eta}\right)^{\alpha} \xi_{s}(t, a, \eta)-\frac{\partial^{\alpha} \eta}{\partial \eta^{\alpha}}\right| \leqq C_{\alpha}|s+t|^{-1-\varepsilon} .
\end{aligned}
$$

Here $\varepsilon=\min (m(l)-l-1: l \leqq|\alpha|)$ and $m(l)=\max (m(\alpha):|\alpha|=l)$.

Proof. Since the case $a \neq 0$ can be treated in a similar way, we prove the case $a=0$ and we omit the variable $a$ in the following expressions. For simplicity we also assume $m=1$. Since $V(x)$ satisfies the Assumption $(\mathrm{A}),\left(\frac{\partial}{\partial \eta}\right)^{\alpha} x_{s}(t, \eta)$ and $\left(\frac{\partial}{\partial \eta}\right)^{\alpha} \xi_{s}(t, \eta)$ satisfy the integral equations

$$
\left.\begin{array}{l}
\left(\frac{\partial}{\partial \eta}\right)^{\alpha} x_{s}(t, \eta)=(s+t) \frac{\partial^{\alpha} \eta}{\partial \eta^{\alpha}}+\int_{0}^{t}(t-u)\left(\frac{\partial}{\partial \eta}\right)^{\alpha}\left(-\operatorname{grad}_{x} V\left(x_{s}(u, \eta)\right)\right) d u \\
\left(\frac{\partial}{\partial \eta}\right)^{\alpha} \xi_{s}(t, \eta)=\frac{\partial^{\alpha} \eta}{\partial \eta^{\alpha}}+\int_{0}^{t}\left(\frac{\partial}{\partial \eta}\right)^{\alpha}\left(-\operatorname{grad}_{x} V\left(x_{s}(u, \eta)\right)\right) d u
\end{array}\right\}
$$

Let us write $F(x)=-\operatorname{grad}_{x} V(x)$. By an elementary calculus we see that $\left(\frac{\partial}{\partial \eta}\right)^{\alpha} F\left(x_{s}(t, \eta)\right)$ is the sum of $\frac{\partial F}{\partial x} \cdot\left(\frac{\partial}{\partial \eta}\right)^{\alpha} x_{s}(t, \eta)$ and the terms of the form

$$
\frac{\partial^{\beta} F}{\partial x^{\beta}}\left(x_{s}(t, \eta)\right) \cdot\left(\frac{\partial^{\sigma_{1}} x}{\partial \eta^{\sigma}{ }_{1}}\right)^{\delta_{1}} \ldots\left(\frac{\partial^{\sigma_{j}} x}{\partial \eta^{\sigma_{J}}}\right)^{\delta_{3}}
$$

where $|\beta| \geqq 2, \beta \leqq \alpha, \delta_{1}+\ldots+\delta_{j}=|\beta|, \sigma_{l}<\alpha \quad$ and $\sum\left|\sigma_{l}\right| \cdot \delta_{l}=|\alpha|$. Let us take as $\varepsilon=\min (m(0)-1, m(1)-2)$ and $R=\sup _{n \in K}\left\{\left(\max \left\{C_{1}, \frac{3}{2} n C_{2}\right\}\right)^{1 /(1+\varepsilon)} \varepsilon^{-1 /(1+\varepsilon)}\right.$ $\left.\cdot|\eta|^{-(3+\varepsilon) /(1+\varepsilon)}(1+|\eta|)\right\}$

where $C_{j}=\max _{|\alpha|=j} C_{\alpha}$ and $C_{\alpha}$ 's are the constants appeared in Assumption (A). We 
first prove (2.4) for the cases $\alpha=0$ and $|\alpha|=1$ then prove the general cases by induction.

(a) The case $\alpha=0$. Put

$$
t^{*}=\sup \left\{0 \leqq t \leqq-s:\left|x_{s}(u, \eta)-(s+u) \eta\right| \leqq 2^{-1}|s+u||\eta| \text { for } 0<u<t\right\} .
$$

It suffices to prove $t^{*} \geqq-s-R$. By Assumption (A) and the definition of $t^{*}$ we get

$$
\begin{aligned}
\left|x_{s}\left(t^{*}, \eta\right)-\left(s+t^{*}\right) \eta\right| & \leqq C_{1} \int_{0}^{t^{*}}\left(t^{*}-u\right)\left(1+\left|x_{s}(u, \eta)\right|\right)^{-2-\varepsilon} d u \\
& \leqq C_{1} \int_{0}^{t^{*}}\left(t^{*}-u\right)\left(2^{-1}|s+u \| \eta|\right)^{-2-\varepsilon} d u \\
& \leqq C_{1} 2^{2+\varepsilon}|\eta|^{-2-\varepsilon} \varepsilon^{-1}\left(-s-t^{*}\right)^{-\varepsilon}
\end{aligned}
$$

Hence if $t^{*}<-s-R$, the right hand side is strictly smaller than $2^{-1}\left(-s-t^{*}\right)|\eta|$ which leads to a contradiction because $x_{s}(t, \eta)$ is continuous in $t$.

(b) The case $|\alpha|=1$. By (2.6) we have the equation for $g_{s}(t, \eta)=\frac{\partial x}{\partial \eta}(t, \eta)-(s+t) e$, $e=\left\{\delta_{i j}\right\}_{i j}$,

$$
g_{s}(t, \eta)=\int_{0}^{t}(t-u) \frac{\partial F}{\partial x}\left(x_{s}(u, \eta)\right)(s+u) e d u+\int_{0}^{t}(t-u) \frac{\partial F}{\partial x}\left(x_{s}(u, \eta)\right) g_{s}(u, \eta) d u
$$

For $0 \leqq t \leqq-s-R$, we get by the result for the case $\alpha=0$ that

$$
\begin{aligned}
& \left|\int_{0}^{t}(t-u) \frac{\partial F}{\partial x}\left(x_{s}(u, \eta)\right)(s+u) e d u\right| \\
& \quad \leqq n C_{2}\left(2^{-1}|\eta|\right)^{-3-\varepsilon} \int_{0}^{t}(t-u)(-s-u)^{-2-\varepsilon} d u \\
& \quad \leqq n C_{2}\left(2^{-1}|\eta|\right)^{-3-\varepsilon} \varepsilon^{-1}(-s-t)^{-\varepsilon} .
\end{aligned}
$$

Let us define as

$$
t^{* *}=\sup \left\{0 \leqq t \leqq-s:\left|g_{s}(u, \eta)\right| \leqq 2^{-1}|s+u| \text { for } 0 \leqq u \leqq t\right\} .
$$

Again it suffices to prove $t^{* *} \geqq-s-R$. By (2.7) we have

$$
\begin{aligned}
\left|g_{s}\left(t^{* *}, \eta\right)\right| \leqq & n C_{2}\left(2^{-1}|\eta|\right)^{-3-\varepsilon}\left\{\varepsilon^{-1}\left(-s-t^{* *}\right)^{-\varepsilon}\right. \\
& \left.+(1 / 2) \int_{0}^{t^{*}}\left(t^{* *}-u\right)(-s-u)^{-3-\varepsilon}(-s-u) d u\right\} \\
\leqq & C_{2} \frac{3 n}{2}\left(2^{-1}|\eta|\right)^{-3-\varepsilon} \varepsilon^{-1}\left(-s-t^{* *}\right)^{-\varepsilon}
\end{aligned}
$$

Hence if $t^{* *}<-s-R$, the right hand side of (2.8) is strictly smaller than $2^{-1}\left|s+t^{* *}\right|$, which again leads to a contradiction. 
(c) The general case. Let us assume that the statement is true for all $\alpha^{\prime}<\alpha, 2 \leqq|\alpha|$. $\left(\frac{\partial}{\partial \eta}\right)^{\alpha} x_{s}(t, \eta)$ satisfies the equation

$$
\begin{aligned}
\left(\frac{\partial}{\partial \eta}\right)^{\alpha} x_{s}(t, \eta)= & \int_{0}^{t}(t-u) \frac{\partial F}{\partial x}\left(x_{s}(u, \eta)\right)\left(\frac{\partial}{\partial \eta}\right)^{\alpha} x_{s}(u, \eta) d u \\
& +\int_{0}^{t}(t-u)\left\{\left(\frac{\partial}{\partial \eta}\right)^{\alpha} F\left(x_{s}(u, \eta)\right)-\frac{\partial F}{\partial x}\left(x_{s}(u, \eta)\right)\left(\frac{\partial}{\partial \eta}\right)^{\alpha} x_{s}(u, \eta)\right\} d u .
\end{aligned}
$$

By the assumption of the induction and the remark preceding part (a), we can easily see that

$$
\begin{aligned}
& \left|\left(\frac{\partial}{\partial \eta}\right)^{\alpha} F\left(x_{s}(u, \eta)\right)-\frac{\partial F}{\partial x}\left(x_{s}(u, \eta)\right)\left(\frac{\partial}{\partial \eta}\right)^{\alpha} x_{s}(u, \eta)\right| \\
& \quad \leqq C|s+u|^{-2-\varepsilon} \text { for } 0 \leqq u \leqq-s-R, \quad \eta \in K .
\end{aligned}
$$

Hence using other constants $M_{1}$ and $M_{2}$, we get

$$
\begin{aligned}
& \left|\left(\frac{\partial}{\partial \eta}\right)^{\alpha} x_{s}(t, \eta)\right| \leqq M_{1}|s+t|^{-\varepsilon}+M_{2} \int_{0}^{t}(t-u)|s+u|^{-2-\varepsilon}\left|\left(\frac{\partial}{\partial \eta}\right)^{\alpha} x_{s}(u, \eta)\right| d u \\
& \leqq M_{1}|s+t|^{-\varepsilon}+M_{2} \int_{0}^{t}|s+u|^{-1-\varepsilon}\left|\left(\frac{\partial}{\partial \eta}\right)^{\alpha} x_{s}(u, \eta)\right| d u .
\end{aligned}
$$

Therefore by Gronwall's inequality we get

$$
\begin{aligned}
\left|\left(\frac{\partial}{\partial \eta}\right)^{\alpha} x_{s}(t, \eta)\right| & \leqq M_{1}(-s-t)^{-\varepsilon}+M_{2} \cdot M_{1} \int_{0}^{t}|s+u|^{-1-2 \varepsilon} e^{C|t+s|^{-\varepsilon}} d u \\
& \leqq M_{1}|s+t|^{-\varepsilon}+M_{3}|s+t|^{-2 \varepsilon} \\
& \leqq M_{4}|s+t|^{-\varepsilon}
\end{aligned}
$$

which proves the general case.

The second relation (2.5) is obvious by virtue of the estimate

$$
\left|\int_{0}^{t}\left(\frac{\partial}{\partial \eta}\right)^{\alpha} F\left(x_{s}(u, \xi)\right) d u\right| \leqq M|s+t|^{-1-\varepsilon} \quad \text { for } 0 \leqq t \leqq-s-R .
$$

Corollary 2.2. For sufficiently large $-s$,

$$
\pi_{j} U(t) U_{0}(s) \pi_{2, a}^{*}, j=1,2 \text { and } 0 \leqq t \leqq-s-R,
$$

is $C^{\infty}$-diffeomorphism from $K$ to its image.

This is a well-known consequence of (2.4) and (2.5) (see also Hörmander [8]).

The following lemma is concerned with the existence of the wave operator in the classical mechanics.

Lemma 2.3. i) For any $a \in \mathbb{R}^{n}$ and $\eta \in K \subset \subset \mathbb{R}^{n} \backslash\{0\}$,

$$
\lim _{s \rightarrow-\infty}\left(\left(\frac{\partial}{\partial \eta}\right)^{\alpha} x_{s}(-s-R, a, \eta), \quad\left(\frac{\partial}{\partial \eta}\right)^{\alpha} \xi_{s}(-s-R, a, \eta)\right)
$$


exists for any multi-index $\alpha$. Furthermore the convergence is uniform on K. Hence

$$
W_{-, R}^{c l}=\lim _{s \rightarrow-\infty} U(-s-R) U_{0}(s)
$$

is a $C^{\infty}$-canonical transformation from $\pi_{2 . a}^{*} K$ to its image.

ii) $\pi_{j} W_{-, R}^{c l} \pi_{2, a}^{*}=\Omega_{j,-}^{R, a}(j=1,2)$ is a $C^{\infty}$-diffeomorphism from $K$ to its image.

Proof. i) is known for $|\alpha| \leqq 1$, see Simon [12] and Herbst [6]. For general $|\alpha| \geqq 2$, we can mimic their proof. ii) is an immediate consequence of i) and Collorary 2.2.

(Q.E.D.)

Remark 2.4. Lemma 2.3 and its proof show that for any $(a, \eta) \in \mathbb{R}^{n} \times\left(\mathbb{R}^{n} \backslash\{0\}\right)$ there exists a solution of (2.3) such that

$$
\lim _{t \rightarrow-\infty}|x(t)-a-t \eta / m|=0, \quad \lim _{t \rightarrow-\infty}|\xi(t)-\eta|=0 .
$$

We write this solution as $\left(x_{-}(t, a, \eta), \xi_{-}(t, a, \eta)\right)$.

The important theorem in the scattering theory of the classical mechanics is the one by Hunziker and Siegel on the completeness of the scattering states. In our context it may be stated as follows (see Hunziker [9] for the details and the proof).

Theorem 2.5. (Hunziker-Siegel) Let Assumption (A) be satisfied. Then there exists a closed null set $e \subset \mathbb{R}^{n} \times \mathbb{R}^{n}$ such that the following statements hold: i) For any $(a, \eta) \in \mathbb{R}^{2 n} \backslash$ e there exists $\left(a_{+}(a, \eta), \eta_{+}(a, \eta)\right) \in \mathbb{R}^{2 n}$ such that the solution $(x(t), \xi(t))=$ $\left(x_{-}(t, a, \eta), \xi_{-}(t, a, \eta)\right)$ satisfies

$$
\lim _{t \rightarrow \infty}\left|x(t)-a_{+}-\eta_{+} t / m\right|=0, \quad \lim _{t \rightarrow \infty}\left|\xi(t)-\eta_{+}\right|=0 .
$$

ii) The mapping $S^{c l}$ defined as $S^{c l}(a, \eta)=\left(a_{+}(a, \eta), \eta_{+}(a, \eta)\right)$ is a $C^{1}$-canonical mapping. Hence by Fubini's theorem, we get the following result:

Corollary 2.6. For any $a \in \mathbb{R}^{n}$, let us define $e(a)=\left\{\eta \in \mathbb{R}^{n}:(a, \eta) \in e\right\} \cup\{0\}$. Then the statement of Theorem 2.5 holds for any $(a, \eta)$ with $\eta \in \mathbb{R}^{n} \backslash e(a)$ and $e(a)$ is null set for almost all $a \in \mathbb{R}^{n}$.

Remark. If $n=1$, we can easily prove that $e(a)$ is closed null set for all $a \in \mathbb{R}^{n}$. We believe that this is true even for $n \geqq 2$. However we could not prove it.

Now we study the asymptotic behavior as $t \rightarrow \infty$ of the solutions of (2.3) which satisfy Theorem 2.5. The study has been intensively done by Hörmander [8] and others [6], [12] in other contexts, however, we need a slightly different estimates.

Lemma 2.7. Let $a \in \mathbb{R}^{n}$ and $K \subset \subset \mathbb{R}^{n} \backslash e(a)$. Then the following statements hold. i) $\eta_{+}(a, \eta)$ and $a_{+}(a, \eta)$ are infinitely differentiable functions of $\eta \in K$.

ii) There exists sufficiently large $t_{0}>0$ such that for any $\alpha>0$, there exists a constant $C_{\alpha}$ independent of $\eta \in K$ such that

$$
\begin{aligned}
& \left|\left(\frac{\partial}{\partial \eta}\right)^{\alpha}\left(x_{-}(t, a, \eta)-\eta_{+}(a, \eta) t / m-a_{+}(a, \eta)\right)\right| \leqq C_{\alpha} t^{-\varepsilon}, \\
& \left|\left(\frac{\partial}{\partial \eta}\right)^{\alpha}\left(\xi_{-}(t, a, \eta)-\eta_{+}(a, \eta)\right)\right| \leqq C_{\alpha} t^{-1-\varepsilon}
\end{aligned}
$$

for $t>t_{0}$. 
Proof. We mimic the argument of Hunziker [9]. We set $m=1$. For $t>0$, let us write as $C_{t}=\left\{\eta \in \mathbb{R}^{n} \backslash e(a): \dot{x}_{-}(t, a, \eta) \cdot x_{-}(t, a, \eta) \geqq(t / 2)|\eta|^{2} \quad\right.$ and $\left.C_{0}\left(1+\left|x_{-}(t, a, \eta)\right|\right)^{-m(0)}+C_{1}\left(1+\left|x_{-}(t, a, \eta)\right|\right)^{-m(1)}\left|x_{-}(t, a, \eta)\right| \leqq 2^{-1}|\eta|^{2}\right\}$ (let us remark $\left|\eta_{+}\right|=|\eta|$ by the conservation of energy). Since for $x(t)=x_{-}(t, a, \eta)$,

$$
2^{-1}(d / d t) x(t)^{2}=x(t) \cdot \dot{x}(t)
$$

and

$$
2^{-1}\left(d^{2} / d t^{2}\right) x(t)^{2}=|\eta|^{2}-\{2 V(x(t))-F(x(t)) \cdot x(t)\},
$$

$C_{t}$ is monotonically increasing in $t>0$ and by Theorem $2.5 \bigcup_{t>0} C_{t}=\mathbb{R}^{n} \backslash e(a)$. Since $\bar{K}$ is compact, there exists a constant $t_{0}>0$ such that $C_{t_{0}} \supset \bar{K}$, which implies the existence of (another) constant $t_{0}$ such that

$$
\left|x_{-}(t, a, \eta)\right| \geqq 2^{-1}|\eta| t, \quad \text { for } \eta \in K \text { and } t>t_{0} .
$$

Now let us note that $x_{-}(t, a, \eta)$ and $\xi_{-}(t, a, \eta)$ satisfy the integral equations (see Simon [12]):

$$
\begin{aligned}
& x_{-}(t, a, \eta)=a_{+}+\eta_{+} t-\int_{t}^{\infty}(t-u) F\left(x_{-}(u, a, \eta)\right) d u, \\
& \xi_{-}(t, a, \eta)=\eta_{+}-\int_{t}^{\infty} F\left(x_{-}(u, a, \eta)\right) d u
\end{aligned}
$$

as well as the equations

$$
\begin{aligned}
& x_{-}(t, a, \eta)=a+t \eta+\int_{-\infty}^{t}(t-u) F\left(x_{-}(u, a, \eta)\right) d u, \\
& \xi_{-}(t, a, \eta)=\eta+\int_{-\infty}^{t} F\left(x_{-}(u, a, \eta)\right) d u .
\end{aligned}
$$

Hence

$$
\begin{aligned}
& a_{+}(a, \eta)=a-\int_{-\infty}^{\infty} u \cdot F\left(x_{-}(u, a, \eta)\right) d u, \\
& \eta_{+}(a, \eta)=\eta+\int_{-\infty}^{\infty} F\left(x_{-}(u, a, \eta)\right) d u .
\end{aligned}
$$

Therefore Lemma 2.1 and 2.11 show the continuity of $a_{+}(a, \eta)$ and $\eta_{+}(a, \eta)$. Now differentiating (2.17) by $\left(\frac{\partial}{\partial \eta}\right)^{\alpha}$ and using the Gronwall's inequality, we can easily see that for any $0 \leqq \alpha,\left|\left(\frac{\partial}{\partial \eta}\right)^{\alpha} x_{-}(t, a, \eta)\right|$ is majorized by $C_{\alpha} t$. This with Lemma 2.1 and with Lebesgue's dominated convergence theorem implies that

$$
\left(\frac{\partial}{\partial \eta}\right)^{\alpha} \int_{-\infty}^{\infty} u F\left(x_{-}(u, a, \eta)\right) d u \text { and }\left(\frac{\partial}{\partial \eta}\right)^{\alpha} \int_{-\infty}^{\infty} F\left(x_{-}(u, a, \eta)\right) d u
$$

exists. This completes the proof for i). 
For proving ii), it suffices to prove (2.13), since (2.12) can be obtained by integrating (2.13). Differentiating (2.16) and noting $\left|\left(\frac{\partial}{\partial \eta}\right)^{\alpha} x_{-}(t, a, \eta)\right| \leqq C_{\alpha} t$, we have

$$
\left(\frac{\partial}{\partial \eta}\right)^{\alpha}\left(\xi_{-}(t, a, \eta)-\eta_{+}\right)=\int_{t}^{\infty}\left(\frac{\partial}{\partial \eta}\right)^{\alpha} F\left(x_{-}(u, a, \eta)\right) d u \text {. }
$$

Since $\left|\left(\frac{\partial}{\partial \eta}\right)^{\alpha} F\left(x_{-}(u, a, \eta)\right)\right| \leqq C|u|^{-2-\varepsilon}$ by virtue of the remark given in the proof of Lemma 2.1, inequality (2.13) follows obviously.

Let us define the scattering operator $S_{a}^{c l}$ with index $a \in \mathbb{R}^{n}$ as

(Q.E.D.)

$$
S_{a}^{c l} \eta=\eta_{+}(a, \eta)
$$

\section{Properties of Action Integrals}

In this section, we shall study several properties of the solutions of the HamiltonJacobi equation

$$
\left(\frac{\partial S}{\partial t}\right)(t, x)+(2 m)^{-1}\left(\operatorname{grad}_{x} S(t, x)\right)^{2}+V(x(t, x))=0 .
$$

We first discuss them in the region $(-\infty, t)$ with $-t$ sufficiently large, using the estimates of Lemma 2.1.

Lemma 3.1. Let $K \subset \subset \mathbb{R}^{n} \backslash\{0\}$ and let $R>0$ be the constant determined in Lemma 2.1. For $-s>R$ let $u s$ define the function $S_{s}(t, x)$ on the domain $\mathscr{Q}_{s, R}(K, a)=$ $\bigcup_{0 \leqq t \leqq-s-R}\{t\} \times \pi_{1} U(t) U_{0}(s) \pi_{2, a}^{*} K \subset \mathbb{R}^{1} \times \mathbb{R}^{n}$ as

$$
S_{s}(t, x)=\frac{s}{2 m} \eta_{s}(t, a, x)^{2}+\int_{0}^{t} L\left(x_{s}\left(u, a, \eta_{s}(t, a, x)\right), \dot{x}_{s}\left(u, a, \eta_{s}(t, a, x)\right) d u,\right.
$$

where $L(x, \dot{x})=m \dot{x}^{2} / 2-V(x)$ is the Lagrangian of the system and $\eta_{s}(t, a, x)=$ $\left(\pi_{1} U(t) U_{0}(s) \pi_{2, a}^{*}\right)^{-1} x$. Then the following statements hold:

i) $S_{s}(t, x)$ is an infinitely differentiable function of $(t, x)$ on $\mathscr{Q}_{s, R}(K, a)$.

ii) $S_{s}(t, x)$ satisfies the Hamilton-Jacobi Eq. (3.1) with the initial condition

$$
S_{s}(0, x)=m(x-a)^{2} / 2 s .
$$

iii) Let us define as

$$
K_{s}(t, x)=-(2 m)^{-1} \int_{0}^{t}\left(\Delta_{x} S_{s}\right)\left(u, x_{s}\left(u, \eta_{s}(t, a, x)\right)\right) d u,
$$

then there exists a constant $C>0$ such that

$$
\begin{aligned}
& \left|\frac{\partial}{\partial y} K(t, x)\right| \leqq C|s|^{-1}|s+t|^{-1-\varepsilon}, \\
& \left|\frac{\partial^{2}}{\partial y^{2}} K(t, x)\right| \leqq C|s|^{-2}|s+t|^{-1-\varepsilon},
\end{aligned}
$$

where $y=s \eta_{s}(t, a, x)+a$. 
iv) For any multi-index $\alpha, \lim _{s \rightarrow-\infty}\left(\frac{\partial}{\partial x}\right)^{\alpha} S_{s}\left(-s-R, x_{s}(-s-R, a, \eta)\right)$ exists uniformly on $K$, and $\lim _{s \rightarrow-\infty}\left(\frac{\partial}{\partial x}\right)^{\alpha} S_{s}(-s-R, x)$ exists on $\Omega_{1,-}^{R, a}(K)$ uniformly. We write as

$$
S_{-\infty}^{R}(x)=\lim _{s \rightarrow-\infty} S_{s}(-s-R, x) .
$$

Proof. Since the case $a \neq 0$ can be treated similarly by changing the variable $x$ to $x-a$, we assume $a=0$ in what follows and omit the variable $a$. We also assume $m=1$. Statement i) is obvious and ii) is well known (see Landau-Lifschitz [15]). Let us prove iii) and iv).

Proof of iii). Since as is well known, $(\partial S / \partial x)\left(u, x_{s}(u)\right)=\xi\left(u, \eta_{s}(t, x)\right), \quad\left(\Delta_{x} S\right)$. $\left(u, x_{s}\left(u, \eta_{s}(t, x)\right)\right)=\sum_{j=1}^{n} \partial \xi_{j} / \partial \bar{x}_{j}=\sum_{j, k=1}^{n}\left(\partial y_{k} / \partial \bar{x}_{j}\right)\left(\partial \xi_{j} / \partial y_{k}\right)$, where $y=s \eta_{s}(t, x)$ and $\bar{x}=$ $x_{s}(u)=x_{s}\left(u, \eta_{s}(t, x)\right)$. Then by elementary calculations, we get

$$
\begin{aligned}
& \left(\partial / \partial y_{m}\right)\left(\Delta_{x} S\right)\left(u, x_{s}\left(u, \eta_{s}(t, x)\right)\right. \\
& =\sum_{j, k, l}\left(\partial \bar{x}_{l} / \partial y_{m}\right)\left(\partial^{2} y_{k} / \partial \bar{x}_{j} \partial \bar{x}_{l}\right)\left(\partial \xi_{j} / \partial y_{k}\right)+\sum_{j, k}\left(\partial y_{k} / \partial \bar{x}_{j}\right)\left(\partial^{2} \xi_{j} / \partial y_{k} \partial y_{m}\right) \\
& \left(\partial^{2} / \partial y_{m} \partial y_{n}\right)\left(\Delta_{x} S\right)\left(u, x_{s}\left(u, \eta_{s}(t, x)\right)\right. \\
& =\sum_{j, k, l}\left(\partial^{2} \bar{x}_{l} / \partial y_{m} \partial y_{n}\right)\left(\partial^{2} y_{k} / \partial \bar{x}_{j} \partial \bar{x}_{l}\right)\left(\partial \xi_{j} / \partial y_{k}\right) \\
& \quad+\sum_{j, k, l, i}\left(\partial \bar{x}_{l} / \partial y_{m}\right)\left(\partial \bar{x}_{i} / \partial y_{n}\right)\left(\partial^{3} y_{k} / \partial \bar{x}_{l} \partial \bar{x}_{j} \partial \bar{x}_{i}\right)\left(\partial \xi_{j} / \partial y_{k}\right) \\
& \quad+\sum_{j, k, l}\left(\partial \bar{x}_{l} / \partial y_{m}\right)\left(\partial^{2} y_{k} / \partial \bar{x}_{l} \partial \bar{x}_{j}\right)\left(\partial^{2} \xi_{j} / \partial y_{k} \partial y_{n}\right) \\
& \quad+\sum_{j, k, l}\left(\partial^{2} y_{k} / \partial \bar{x}_{j} \partial \bar{x}_{l}\right)\left(\partial \bar{x}_{l} / \partial y_{n}\right)\left(\partial^{2} \xi_{j} / \partial y_{k} \partial y_{m}\right) \\
& \quad+\sum_{j, k}\left(\partial y_{k} / \partial \bar{x}_{j}\right)\left(\partial^{3} \xi_{j} / \partial y_{k} \partial y_{m} \partial y_{n}\right) .
\end{aligned}
$$

Since $\bar{x}=x_{s}(u, y / s)$, Lemma 2.1 and simple calculations show that for $y=s \eta$ and $\bar{x}=x_{s}(u, \eta)$

$$
\begin{aligned}
& |(\partial y / \partial x)| \leqq C|s||s+u|^{-1} \\
& \left|\left(\partial^{\alpha} y / \partial x^{\alpha}\right)\right| \leqq C_{\alpha}|s||s+u|^{-1-\varepsilon-|\alpha|}, \quad|\alpha| \geqq 2 .
\end{aligned}
$$

Thus using (3.9) and Lemma 2.1, we get from (3.7) and (3.8) the estimates

$$
\left|\left(\partial / \partial y_{k}\right)\left(\Delta_{x} S\right)(u, x(u))\right| \leqq C|s|^{-1}|s+u|^{-2-\varepsilon}
$$

and

$$
\left|\left(\partial^{2} / \partial y_{n} \partial y_{m}\right)\left(\Delta_{x} S\right)(u, x(u))\right| \leqq C|s|^{-2}|s+u|^{-2-\varepsilon}
$$

from which the desired results (3.5) and (3.6) follow by integration. 
Proof of $i v$ ). We prove only the case $\alpha=0$. Other cases can be proved similarly. By the definition of $S_{s}(t, x)$ and the law of conservation of energy, we have

$$
\begin{aligned}
S_{s}(t, x)= & t\left\{\eta_{s}(t, x)^{2} / 2 m+V\left(s \eta_{s}(t, x)\right)\right\} \\
& -2 \int_{0}^{t} V\left(x_{s}\left(u, \eta_{s}(t, x)\right)\right) d u+\left(s \eta_{s}(t, x)\right)^{2} / 2 s m \\
= & (s+t) \eta_{s}(t, x)^{2} / 2 m+t V\left(s \eta_{s}(t, x)\right)-2 \int_{0}^{t} V\left(x_{s}\left(u, \eta_{s}(t, x)\right)\right) d u .
\end{aligned}
$$

Therefore

$$
\begin{aligned}
S_{s}(-s-R, x)= & -R \eta_{s}(-s-R, x)^{2} / 2 m+(-s-R) V\left(s \eta_{s}(-s-R, x)\right) \\
& -2 \int_{0}^{-s-R} V\left(x_{s}\left(u, \eta_{s}(-s-R, x)\right) d u .\right.
\end{aligned}
$$

Take $K_{1}$ such that $K \subset \subset K_{1} \subset \subset \mathbb{R}^{n} \backslash\{0\}$. For $x \in \Omega_{1,-}^{R}(K), \eta_{s}(-s-R, x) \in K_{1}$ for sufficiently large $-s . \quad \lim \eta(-s-R, x)$ exists and $\lim (-s-R) V \times$ $\left(s \eta_{s}(-s-R, x)\right)=0$ uniformly on $K$, since $\left(\pi_{1} U(-s-R) U_{0}(s) \pi_{2, a}^{s \rightarrow \infty}\right)^{-1}$ converges uniformly on $\Omega_{1,-}^{R}(K)$. Now Lemma 2.1 and Lebesgue's dominated convergence theorem imply the uniform convergence of the integral $\int_{0}^{s-R} V\left(x_{s}\left(u, \eta_{s}(-s-R, x)\right) d u\right.$ as $s \rightarrow-\infty$. This completes the proof.

(Q.E.D.)

Remark 3.2. We remark here that for $x=\Omega_{1,-}^{R . a}(\eta)$,

$$
S_{-\infty}^{R}(x)=\lim _{s \rightarrow-\infty}\left\{\int_{s}^{-R} L\left(x_{-}(u, a, \eta), \quad \dot{x}_{-}(u, a, \eta)\right) d u+\frac{\eta^{2}}{2 m} s\right\} .
$$

Now we turn to the study of the solution of (3.1) in the region $(t, \infty)$ with $t>0$ sufficiently large. We take $K \subset \subset \mathbb{R}^{n} \backslash e(a)^{e x}$ and take $K_{1}$ such that $K \subset \subset K_{1} \subset \subset$ $\mathbb{R}^{n} \backslash e(a)^{e x}$. Then obviously there exists a constant $\delta_{K_{1}}>0$ such that

$\mid \operatorname{det}\left(\partial \eta_{+}(a, \eta) / \partial \eta \mid \geqq \delta_{K_{1}}, \quad \eta \in K_{1}\right.$.

Therefore the mapping $S_{a}^{c l} \eta=\eta_{+}(a, \eta)$ is a locally uniform diffeomorphism on $K_{1}$ and for any $\eta_{+} \in S_{a}^{c l} K$, there exists a small neighbourhood $U_{+}\left(\eta_{+}\right) \subset S_{a}^{c l} K_{1}$ such that the inverse image $\left(S_{a}^{c l}\right)^{-1} U_{+}\left(\eta_{+}\right)$consists of a finite number of disjoint components $\left\{U_{k}\left(\eta_{+}\right)\right\}$on each of which $S_{a}^{c l}$ is diffeomorphism. Thanks to the property (2.12), the correspondence from $\eta_{+}(a, \eta)$ to $x_{-}(t, a, \eta)$ is diffeomorphism for sufficiently large $t>t_{0}, t_{0}$ is determined only by $K$. So that the mapping $x_{-}(t, a, \cdot)$ for $t>t_{0}$ is also diffeomorphic on $U_{k}\left(\eta_{+}\right)$. Let us take such $U_{k}\left(\eta_{+}\right)$for each $\eta_{+} \in S_{a}^{c l} K$. Obviously $\left\{U_{k}\left(\eta_{+}\right), \eta_{+} \in S_{a}^{c l} K\right\}$ forms an open covering of $K$. Then a simple compactness argument shows there exists a finite number of relatively compact open covering $\left\{U_{k}\right\}$ of $K$ such that $U_{k} \subset K_{1}$ and on each $U_{k}, S_{a}^{c l}$ and the mapping $x_{-}(t, a, \cdot)\left(t>t_{0}\right)$ are diffeomorphisms. Let us take one of these $U_{k}$ 's and label it as $U$. 
Lemma 3. 3. Let Assumption $(A)$ be satisfied. Let $K \subset \subset K_{1} \subset \subset \mathbb{R} \backslash e(a)^{e x}$. Let us take $U$ and $t_{0}>0$ as in the preceding remark and define the function $S(t, x)$ on the domain $2\left(t_{0}, U\right)=\bigcup_{t \geqq t_{0}}\{t\} \times \pi_{1} U(t+R) W_{-, R}^{c l} \pi_{2, a}^{*} U$ as

$$
\begin{aligned}
S(t, x)= & \int_{-R}^{t} L\left(x_{-}(u, a, \eta(t, a, x)), \quad \dot{x}_{-}(u, a, \eta(t, a, x)) d u\right. \\
& +S_{-\infty}^{R}\left(x_{-}(-R, a, \eta(t, a, x)) .\right.
\end{aligned}
$$

where $\eta(t, a, x) \in U$ is determined by the relation $x=x_{-}(t, a, \eta(t, a, x))$. Then the following statements hold:

i) $S(t, x)$ is an infinitely differentiable function on $2\left(t_{0}, U\right)$.

ii) $S(t, x)$ satisfies the Hamilton-Jacobi equation (3.1) on the domain $\mathscr{Z}\left(t_{0}, U\right)$.

iii) $(\partial S / \partial x)(t, x)=\xi_{-}(t, a, \eta(t, a, x))$.

iv) Let $u$ s define the function $K_{t_{0}}(t, x)$ on the domain $2\left(t_{0}, U\right)$ as

$$
K_{t_{0}}(t, x)=-(2 m)^{-1} \int_{t_{0}}^{t}\left(\Delta_{x} S\right)\left(u, x_{-}(u, a, \eta(t, a, x))\right) d u .
$$

Then for any multi-index $\alpha$ there exists a constant $C_{\alpha}>0$ such that

$$
\left|(\partial / \partial y)^{\alpha} K_{t_{0}}(t, x)\right| \leqq C_{\alpha} \log t
$$

where $y=x_{-}\left(t_{0}, \eta(t, a, x)\right)$.

Proof. Statements i), ii) and iii) can be easily checked. We prove iv) only. As in the proof of Lemma 3.1, we can easily get the following estimates from Lemma 2.7 and (3.11):

$$
\begin{aligned}
& \left|(\partial / \partial x)^{\alpha} y(t, x)\right| \leqq C_{\alpha} t^{-|\alpha|}, \quad t \geqq t_{0}, \quad|\alpha| \geqq 1 ; \\
& \left|(\partial / \partial y)^{\alpha} x\left(t, \eta\left(t_{0}, a, y\right)\right)\right| \leqq C_{\alpha} t, \quad t \geqq t_{0}, \quad|\alpha| \geqq 1 ; \\
& \left|(\partial / \partial y)^{\alpha}\left(\xi_{-}\left(t, \eta\left(t_{0}, a, y\right)\right)\right)\right| \leqq C_{\alpha}, \quad t \geqq t_{0},|\alpha| \geqq 1 ;
\end{aligned}
$$

Since $(\partial S / \partial x)(t, x)=\xi_{-}(t)$, we can use the formulas (3.7) and (3.8), and after a simple calculation we get

$$
\left|(\partial / \partial y)^{\alpha}\left(\Delta_{x} S\right)\left(y, a, x_{-}(u, a, \eta(t, a, x))\right)\right| \leqq C_{\alpha} u^{-1},
$$

for any $u \geqq t_{0}$ and multi-index $\alpha$, from which the desired estimates follow by integration.

(Q.E.D.)

\section{The Asymptotic Behavior of the Wave Operator $W_{-}^{h}$}

In this section we study the asymptotic behavior of the wave operator $W_{-}^{h}$ on the coherent state $\tilde{f}_{a}^{h}$. The crucial step of the study is the following theorem.

Theorem 4.1. Let Assumption $(A)$ be satisfied. Let $K \subset \subset \mathbb{R} \mathbb{R}^{n} \backslash\{0\}$ and $R$ be the constant determined in Lemma 2.1. For $f \in \mathscr{H}$ with $\operatorname{supp} f \subset K$, let us define the function $f_{a, s}(t, x)$ for $t \leqq-s-R$ as

$$
f_{a, s}(t, x)=(m / i s)^{n / 2} f\left(\eta_{s}(t, a, x)\right) e^{K_{s}(t, x)} .
$$


Then

$$
\lim _{h \downarrow 0} \sup _{-s \geqq R} \sup _{0 \leqq t \leqq-s-R}\left\|e^{-i t H h / \hbar} T_{0, a}^{h}(s) f-e^{i S_{s}(t, x) / \hbar} f_{a, s}(t, x)\right\|=0 .
$$

Proof. For simplicity we assume $a=0$ and $m=1$. First of all we remark that by the well known result $\exp (2 K(t, x)) d x=d y$, for $y=s \eta_{s}(t, a, x)$ (see for example Maslov [11] ), we easily see $\left\|f_{s}(t, x)\right\|=\|f\|$. Since $\exp \left(-i t H^{h} / \hbar\right) T_{0}^{h}$ is also unitary, it suffices to prove (4.2) for $f \in C_{0}^{\infty}(K)$. Since, as can be easily checked, $f_{s}(t, x)$ satisfies the transport equation

$$
\frac{\partial}{\partial t} f_{s}(t, x)+\sum_{k=1}^{n}\left(\frac{\partial S}{\partial x_{k}}\right)\left(\frac{\partial}{\partial x}\right) f_{s}(t, x)+2^{-1}\left(\Delta_{x} S\right)(t, x) f_{s}(t, x)=0,
$$

we get

$$
\left(i \hbar \frac{\partial}{\partial t}+\frac{\hbar^{2}}{2} \Delta-V(x)\right) e^{i S_{s}(t, x) / \hbar} f_{s}(t, x)=2^{-1} \hbar^{2} e^{i S_{s}(t, x) / \hbar}\left(\Delta_{x} f_{s}\right)(t, x) .
$$

Since $\exp \left(i S_{s}(0, x) / \hbar\right) \cdot f_{s}(0, x)=T_{0}^{h}(s) \cdot f$, we get by (4.4) and Duhamel's principle that

$$
\begin{aligned}
& \left\|e^{-i t H^{h / \hbar}} T_{0}^{h}(s) f-e^{i S_{s}(t, x) / \hbar} f_{s}(t, x)\right\| \\
& \quad \leqq(\hbar / 2)\left\|\int_{0}^{t} e^{-i(t-u) H^{h / \hbar}}\left(e^{i S_{s}(u, x) / \hbar}\left(\Delta f_{s}\right)(u, x)\right) d u\right\| \\
& \quad \leqq(\hbar / 2) \int_{0}^{t}\left\|\left(\Delta_{x} f_{s}\right)(u, x)\right\| d u .
\end{aligned}
$$

In what follows we omit the index s. Let us write $s \eta_{s}(u, x)=y(u, x)$ and $g(y / s)=$ $s^{-n / 2} f(y / s)$.

Then

$$
\begin{aligned}
\Delta_{x} f(u, x)= & \Delta_{x}\left(e^{K(u, x)} g(y / s)\right) \\
= & \sum_{k}\left(\Delta_{x} y_{k}\right)\left\{\left(\partial K / \partial y_{k}\right) g(y / s)+s^{-1}\left(\partial g / \partial y_{k}\right)(y / s)\right\} e^{K(u, x)} \\
& +\sum_{k, j, i}\left(\partial y_{k} / \partial x_{j}\right)\left(\partial y_{i} / \partial x_{j}\right)\left[\left\{\partial K / \partial y_{k}\right)\left(\partial K / \partial y_{i}\right)+\left(\partial^{2} K / \partial y_{k} \partial y_{i}\right) g(y / s)\right\} \\
& \left.+s^{-1}\left\{\left(\partial g / \partial y_{k}\right)(y / s) \cdot\left(\partial K / \partial y_{i}\right)+\left(\partial g / \partial y_{i}\right) \partial K / \partial y_{k}\right)\right\} \\
& \left.+s^{-2}\left(\partial^{2} g / \partial y_{k} \partial y_{i}\right)(y / s)\right] e^{K(u, x)}
\end{aligned}
$$

Now remembering that $\left\|\exp (K(u, x)) s^{-n / 2} h(y / s)\right\|=\|h\|$ for any $h \in L^{2}(K)$ by the preceding remark, we get by Lemma 3.1 and (3.9) that

$$
\begin{aligned}
& \left\|\Delta_{x} f(u, x)\right\| \leqq C|s \| s+u|^{-3-\varepsilon}\left\{|s|^{-1}|s+u|^{-1-\varepsilon}\|f\|+|s|^{-1}\|f\|_{1}\right\} \\
& \quad+|s|^{2}|s+u|^{-2}\left\{\left(|s|^{-2}|s+t|^{-2-\varepsilon}+|s|^{-2}|s+t|^{-1-\varepsilon}\|f\|_{1}+s^{-2}\|f\|_{2}\right\}\right. \\
& \quad \leqq C|s+t|^{-2}\|f\|_{2} .
\end{aligned}
$$

Therefore for $0 \leqq t \leqq|s+R|$,

$$
(\hbar / 2) \int_{0}^{t}\|\Delta f(u, x)\| d u \leqq C h\|f\|_{2} \int_{0}^{t}|s+u|^{-2} d u
$$




$$
\leqq C h\|f\|_{2}|s+t|^{-1} \leqq C h\|f\|_{2} R^{-1},
$$

which proves the desired result.

Now we can state and prove the main theorem in this section.

Theorem 4.2. Let Assumption $(A)$ be satisfied. Let $K \subset \subset \mathbb{R}^{n} \backslash\{0\}$ and $f \in \mathscr{H}$ have supp $f \subset K$. Let us define as

$$
\left(W_{-, R}^{a} f\right)(x)=e^{i S_{-x}^{R}(x) / \hbar-i n \pi / 4}\left[\operatorname{det}\left(\partial \Omega_{1,-}^{R, a}(\eta) /\left.\partial(\eta)\right|_{\Omega_{1,-}^{R, a}(\eta)=x}\right]^{-(1 / 2)} f\left(\left(\Omega_{1,-}^{R, a}\right)^{-1} x\right) .\right.
$$

Then

$$
\lim _{h 0}\left\|e^{i R H^{h} / \hbar} W_{-}^{h} \tilde{f}_{a}^{h}-W_{-, R}^{a} f\right\|=0
$$

Proof. From (4.2) we get

$$
0=\lim _{h \downarrow 0} \lim _{s \rightarrow-\infty}\left\|e^{i(s+R) H^{h} / \hbar} e^{-i s H_{0}^{h / h}} \tilde{f}_{a}^{h}-e^{i S_{s}(-s-R, x) / \hbar} f_{a, s}(-s-R, x)\right\| .
$$

The first summand in the right of (4.9) converges to $\exp \left(i R H^{h} / \hbar\right) W_{-}^{h} \tilde{f}_{a}^{h}$ and the second to $W_{-, R}^{a} f$ as $s \rightarrow-\infty$ by virtue of Lemma 2.3, Lemma $3.1 \mathrm{iv)}$ and the relation $\exp (2 K(t, x)) d x=d y, y=s \eta_{s}(t, a, x)$. This proves the theorem. (Q.E.D.)

\section{Approximate Fundamental Solution and the Stationary Phase Method}

Continuing the approximation scheme, we study here the asymptotic behavior of the solution $e^{-i t H^{h} / \hbar} W_{-}^{h} \tilde{f}_{a}^{h}$ as $h \rightarrow 0$ for sufficiently large $t>0$. The fundamental tools in this section and the next section are the approximate fundamental solution of the Schrödinger operator due to Fujiwara [3], the $L^{2}$-boundedness theorem for some oscillatory integral operators by Asada-Fujiwara [2] and the stationary phase method. We first review results of Fujiwara [3] and Asada-Fujiwara [2].

Theorem 5.1. (Fujiwara). Let $V(x)$ be a real valued infinitely differentiable function on $\mathbb{R}^{n}$ satisfying the condition

(C) $\left|\left(\frac{\partial}{\partial x}\right)^{\alpha} V(x)\right| \leqq C_{\alpha}$ for $|\alpha| \geqq 2$.

Then there exists a constant $\delta>0$ such that the following statements hold:

i) For any $t \in[-\delta, \delta]$ and $x, y \in \mathbb{R}^{n}$, there exists a unique solution of the equation of the classical motion (2.3) such that $x(0)=y, x(t)=x$.

ii) Let $S(t, x, y)=\int_{0}^{t} L(x(u, y), \dot{x}(u, y)) d u$ be the action integral along the classical orbit and let $\phi(t, x, y)=t S(t, x, y)$. Then

$$
\begin{aligned}
& \phi(t, x, y) \in C^{\infty}\left((-\delta, \delta) \times \mathbb{R}^{n} \times \mathbb{R}^{n}\right), \\
& \left|(\partial / \partial x)^{\alpha}(\partial / \partial y)^{\beta} \phi(t, x, y)\right| \leqq C_{\alpha \beta} \quad \text { if }|\alpha|+|\beta| \geqq 2 . \\
& \left|\operatorname{det}\left(\partial^{2} \phi / \partial x \partial y\right)(t, x, y)\right| \geqq 2^{-1}, \\
& \left|(\partial / \partial x)^{\alpha}(\partial / \partial y)^{\beta}\left(\Delta_{x} \phi-n m\right)\right| \leqq C_{\alpha \beta} t,
\end{aligned}
$$

where $\left(\partial^{2} \phi / \partial x \partial y\right)$ in (5.3) is the matrix $\left\{\partial^{2} \phi / \partial x_{j} \partial y_{k}\right\}_{J, k}$. 
iii) Define as

$$
\begin{aligned}
a(t, x, y)= & e^{-i n \pi / 4}(m / 2 \pi|t|)^{n / 2} \\
& \cdot \exp \left(-\frac{1}{2} \int_{0}^{t}\left(m^{-1} \Delta_{x} S(u, x(u), y)-n / m\right) d u\right) .
\end{aligned}
$$

Then the function $\left(\frac{|t|}{m}\right)^{n / 2} a(t, x, y)$ is an infinitely differentiable function on $(-\delta, \delta) \times$ $\mathbb{R}^{n} \times \mathbb{R}^{n}$ satisfying the following conditions:

$$
\begin{aligned}
& \left|(\partial / \partial x)^{\alpha}(\partial / \partial y)^{\beta} a(t, x, y)\right| \leqq C_{\alpha \beta}|t|^{n / 2}, \\
& \left|(\partial / \partial x)^{\alpha}(\partial / \partial y)^{\beta} \Delta_{x} a(t, x, y)\right| \leqq C_{\alpha \beta}|t|^{n / 2+1} .
\end{aligned}
$$

iv) Let $E(t, h)$ be the integral operator $(t \in[-\delta, \delta])$ defined as

$$
E(t, h) f(x)=h^{-n / 2} \int_{\mathbb{R}^{n}} e^{i S(t, x, y) / \hbar} a(t, x, y) f(y) d y, \text { for } f \in C_{0}^{\infty}\left(\mathbb{R}^{n}\right) .
$$

Then the operator $E(t, h)$ can be extended to a bounded operator on $\mathscr{H}$ and statifies

$$
\begin{aligned}
& \|E(t, h)\| \leqq C, \quad-\delta \leqq t \leqq \delta \\
& s-\lim _{t \rightarrow 0} E(t, h)=I, I \text { is the identity operator. }
\end{aligned}
$$

v) Let $T>0$ and $0=t_{0}<t_{1}<\ldots<t_{N}=T$ be an arbitrary subdivision of $[0, T]$ such that $\delta(\Delta)=\max _{j}\left|t_{j+1}-t_{j}\right|<\delta$. Then for $0<h \leqq 1$,

$$
\left\|E\left(t_{N}-t_{N-1}, h\right) \ldots E\left(t_{1}-t_{0}, h\right)-e^{-i t H^{h} / \hbar}\right\| \leqq C_{T, \Delta} h
$$

and moreover

$$
\lim _{\delta(\Delta) \rightarrow 0}\left\|E\left(t_{N}-t_{N-1}, h\right) \ldots E\left(t_{1}-t_{0}\right)-e^{-i t H^{h} / h}\right\|=0 .
$$

For the proof and the details, see Fujiwara [3].

The following theorem on the boundedness of certain integral operators due to Asada-Fujiwara [2] also turns out to be quite useful.

Theorem 5.2. (Asada-Fujiwara) Let $\phi(x, \theta, y)$ be a real-valued infinitely differentiable function on $\mathbb{R}^{n} \times \mathbb{R}^{m} \times \mathbb{R}^{n}$ (the case $m=0$ is not excluded) such that

$$
\left|(\partial / \partial x)^{\alpha}(\partial / \partial \theta)^{\beta}(\partial / \partial y)^{\sigma} \phi(x, \theta, y)\right| \leqq C_{\alpha \beta \sigma} \quad \text { if }|\alpha|+|\beta|+|\sigma| \geqq 2,
$$

there exists a constant $\delta>0$ such that

$$
\left|\operatorname{det}\left[\begin{array}{ll}
\left(\partial^{2} \phi / \partial x \partial y\right) & \left(\partial^{2} \phi / \partial x \partial \theta\right) \\
\left(\partial^{2} \phi / \partial \theta \partial y\right) & \left(\partial^{2} \phi / \partial \theta \partial \theta\right)
\end{array}\right]\right| \geqq \delta>0,
$$

and let $a(x, \theta, y) \in \mathscr{B}\left(\mathbb{R}^{n} \times \mathbb{R}^{m} \times \mathbb{R}^{n}\right)$. Let us define the integral operator $A(\gamma)$ as

$$
A(\gamma) f(x)=\gamma^{n / 2} \int e^{i \gamma \phi(x, \theta, y)} a(x, \theta, y) f(y) d y d \theta .
$$

Then there exists a constant $C>0$ determined by a finite number of $C_{\alpha \beta \sigma}$ 's and $\delta>0$ such that

$$
\|A(\gamma) f\| \leqq C\|f\| \text { for } \gamma \geqq 1 .
$$


Furthermore if $a \equiv 0$ in a neighbourhood of $C_{\phi}$ :

$$
C_{\phi}=\left\{(x, \theta, y) \in \mathbb{R}^{n} \times \mathbb{R}^{m} \times \mathbb{R}^{n}: \partial \phi / \partial \theta=0\right\},
$$

then for any $M>0$, there exists a constant $C_{M}>0$ such that

$$
\|A(\gamma) f\| \leqq C_{M} \gamma^{-M}\|f\|, \quad|\gamma| \geqq 1 \text {. }
$$

See Asada-Fujiwara [2] for the proof, the rigorous definition of the integral (5.15) and the details.

For $a \in \mathbb{R}^{n}$, let us take the domain $U \subset \subset \mathbb{R}^{n} \backslash e(a)^{e x}$ and $t_{0} \in \mathbb{R}^{1}$ as in Lemma 3.3. Let $f \in \mathscr{H}$ have supp $f \subset U$. We study the asymptotic behavior of $\exp \left(-i\left(t_{0}+\right.\right.$ $\left.R) H^{h} / \hbar\right) W_{-}^{h} \tilde{f}_{a}^{h}$. We write as $T=t_{0}+R$. Choose $N$ sufficiently large such that $T / N=\tau<\delta$, where $\delta$ is the constant appeared in Theorem 5.1. By Theorem 5.1 and Theorem 4.2 we have

$$
\begin{aligned}
& \lim _{h \downarrow 0}\left\|e^{-i t_{0} H^{h} / h} W_{-}^{h} \tilde{f}_{a}^{h}-e^{-i T H^{h} / h} W_{-, a}^{R} f\right\| \\
& \quad=\lim _{h \downarrow 0}\left\|e^{-i t_{0} H^{h} / h} W_{-}^{h} \tilde{f}_{a}^{h}-E(\tau, h)^{N} W_{-, a}^{R} f\right\|=0 .
\end{aligned}
$$

We write as

$$
\begin{aligned}
& W_{-, a}^{R} f(x)=e^{i S_{-\alpha}^{R}(x) / \hbar} h_{R}(x), \\
& h_{R}(x)=\left.\left|\operatorname{det}\left(\Omega_{1,-}^{R}(\eta) / \partial \eta\right)\right|_{x=\Omega_{1,-}^{R, a}(\eta)}\right|^{-1 / 2} e^{-i n \pi / 4} f\left(\left(\Omega_{1,-}^{R, a}\right)^{-1} x\right) .
\end{aligned}
$$

Then

$$
\begin{aligned}
& E(\tau, h)^{N} W_{-, a}^{R} f(x)=h^{-N n / 2} \int_{\mathbb{R}^{N n}} \exp \left(i \hbar^{-1} \sum_{j=1}^{N} S\left(\tau, x_{j}, x_{j-1}\right)\right) \\
& \prod_{j=1}^{N} a\left(\tau, x_{j}, x_{j-1}\right) \exp \left(i \hbar^{-1} S_{-\infty}^{R}\left(x_{0}\right)\right) h_{R}\left(x_{0}\right) d x_{0} \ldots d x_{N-1},
\end{aligned}
$$

where $x_{0}=y, x_{N}=x$. Let us write $\theta=\left(x_{N-1}, \ldots, x_{1}\right)$ and $\phi_{R, t_{0}}(x, \theta, y)=$ $\sum_{j=1}^{N} S\left(\tau, x_{j}, x_{j-1}\right)+S_{-\infty}^{R}\left(x_{0}\right)$. We want to apply the stationary phase method to the expression (5.21). For each $x \in \mathbb{R}^{n}$, the point of stationary phase $(\theta, y)$ is determined by the equation

$$
d_{(\theta, y)} \phi(x, \theta, y)=0 \text {, }
$$

which is equivalent to the system of equations

$$
\begin{aligned}
& \partial S / \partial x_{N-1}\left(\tau, x, x_{N-1}\right)+\partial S / \partial x_{N-1}\left(\tau, x_{N-1}, x_{N-2}\right)=0, \\
& \ldots \ldots \ldots \ldots \ldots \ldots \ldots \ldots \ldots \ldots \ldots \ldots \ldots \ldots \ldots \ldots \ldots \ldots \ldots \ldots \ldots \ldots \ldots \ldots \ldots \ldots \ldots \ldots \\
& \partial S / \partial x_{1}\left(\tau, x_{2}, x_{1}\right)+\partial S / \partial x_{1}\left(\tau, x_{1}, x_{0}\right)=0, \\
& \partial S / \partial x_{0}\left(\tau, x_{1}, x_{0}\right)+\partial S_{-\infty}^{R}\left(x_{0}\right) / \partial x_{0}=0 .
\end{aligned}
$$

Lemma 5.3. For each $x \in \pi_{1} U(T) W_{-, R}^{c l} \pi_{2, a}^{*}(U)$, there exists a unique $(\theta, y)=$ $\left(x_{N-1}, \ldots, x_{1}, y\right) \in \mathbb{R}^{N n}$ such that $(\theta, y)$ satisfies the equation $(5.22)$ and such that $y \in \pi_{1} W_{-, R}^{c l} \pi_{2, a}^{*}(U)$, and for $x \notin \pi_{1} U(T) W_{-, R}^{c l} \pi_{2, a}^{*}(U)$ there is no point of stationary phase such that $y \in \pi_{1} W_{-, R}^{c l} \pi_{2, a}^{*}(U)$. 
Proof. Since (a) $-\partial S / \partial y(\tau, x, y)$ and $\partial S / \partial x(\tau, x, y)$ are the initial and final momenta of the particle which starts at the point $y$ at time zero and reaches the point $x$ at time $\tau$; (b) $\partial S_{-\infty}^{R}(y) / \partial y=\xi_{-}(-R, a, \eta(-R, a, y))$; (c) the mapping $U \ni \eta \rightarrow$ $x\left(t_{0}, a, \eta\right)$ is diffeomorphism; (d) for any fixed $y \in \mathbb{R}^{n}$ the mapping $\mathbb{R}^{n} \ni x \rightarrow$ $\partial S / \partial y(\tau, x, y) \in \mathbb{R}^{n}$ is a global diffeomorphism by virtue of (5.2) and (5.3), we can conclude that for any $x_{0} \in \pi_{1} W_{-, R}^{c l} \pi_{2, a}^{*}(U)$, the solution of (5.23), considering $\left(x, x_{N-1}, \ldots, x_{1}\right)$ as an unknown variable and $x_{0}=y$ as a parameter, is determined uniquely as $x_{1}=x_{-}\left(-R+\tau, a, \eta\left(-R, a, x_{0}\right)\right), \ldots, x_{N-1}=x_{-}(-R+(N-1) \tau$, $\left.a, \eta\left(-R, a, x_{0}\right)\right)$ and $x=x_{N}=x_{-}\left(-R+N \tau, a, \eta\left(-R, a, x_{0}\right)\right)=x_{-}\left(t_{0}, a, \eta\left(t_{0}, a, x\right)\right)$. By virtue of Lemma 3.3 the mapping $\Omega_{1,-}^{R, a}(U) \ni x_{-}(-R, a, \eta) \rightarrow x_{-}\left(t_{0}, a, \eta\right) \in$ $\pi_{1} U(T) W_{-, R}^{c l} \pi_{2, a}^{*}(U)$ is a diffeomorphism, this implies the lemma.

Let us define $C_{\phi}(U)$ as

$$
C_{\phi}(U)=\left\{(x, \theta, y) \in \mathbb{R}^{n} \times \mathbb{R}^{(N-1) n} \times \mathbb{R}^{n} ; d_{(\theta, y)} \phi(x, \theta, y)=0, y \in \Omega_{-, a}^{R}(U)\right\}
$$

and the mapping $i: C_{\phi}(U) \rightarrow i\left(C_{\phi}(U)\right) \equiv \Lambda_{\phi}(U)$ as $i(x, \theta, y)=(x, \partial \phi / \partial x(x, \theta, y))=$ $\left(x, \xi_{-}\left(t_{0}, a, \eta\left(t_{0}, a, x\right)\right)\right)$. Then we have the following lemma.

Lemma 5.4. $C_{\phi}(U)$ is $n$-dimensional $C^{\infty}$-manifold immersed in $\mathbb{R}^{(N+1) n}$ and is precompact.

Proof. Since $C_{\phi}(U)$ is determined by the equation $d_{(\theta, y)} \phi(x, \theta, y)=0$, for proving the first statement it suffices to prove that $d\left(\partial \phi / \partial \theta_{1}\right), \ldots, d\left(\partial \phi / \partial \theta_{(N-1) n}\right)$, $d\left(\partial \phi / \partial y_{1}\right), \ldots, d\left(\partial \phi / \partial y_{n}\right)$ are linearly independent at each point $(x, \theta, y) \in C_{\phi}(U)$ as elements of $T^{*} \mathbb{R}^{n(N+1)}$, that is,

$$
\operatorname{rank}\left[\begin{array}{lll}
\partial^{2} \phi / \partial x \partial y & \partial^{2} \phi / \partial y \partial y & \partial^{2} \phi / \partial y \partial \theta \\
\partial^{2} \phi / \partial x \partial \theta & \partial^{2} \phi / \partial y \partial \theta & \partial^{2} \phi / \partial \theta \partial \theta
\end{array}\right]=n N
$$

Since $S(\tau, x, y)$ and $S(\tau, x, y)+S_{-\infty}^{R}(y)$ satisfy the condition (5.3), the remark of Asada-Fujiwara [2] (at the end of Sect. 2 of [2]) shows

$$
\operatorname{det}\left[\begin{array}{ll}
\partial^{2} \phi / \partial x \partial y & \partial^{2} \phi / \partial \theta \partial x \\
\partial^{2} \phi / \partial \theta \partial y & \partial^{2} \phi / \partial \theta \partial \theta
\end{array}\right] \neq 0
$$

at each point $(x, \theta, y) \in C_{\phi}(U)$, which obviously implies (5.25). The second statement is obvious.

(Q.E.D.)

Lemma 5.5. The mapping $i: C_{\phi}(U) \rightarrow \Lambda_{\phi}(U)$ is Lagrangian immersion. The projection

$$
\pi_{1}: \Lambda_{\phi}(U) \ni(x, \partial \phi / \partial x) \rightarrow x \in \pi_{1}\left(\Lambda_{\phi}(U)\right)
$$

is a diffeomorphism.

Proof. The first statement is proved by Hörmander [7] (see also Asada-Fujiwara [2]). The second is obvious by Lemma 3.3.

(Q.E.D.)

Lemma 5.6. There exists a constant $\kappa>0$ such that on $C_{\phi}(U)$, $\left|\operatorname{det} \operatorname{Hess}_{(\theta . y)}(\phi)(x, \theta, y)\right| \geqq \kappa>0$, 


$$
\operatorname{Hess}_{(\theta, y)}(\phi)=\left[\begin{array}{ll}
\partial^{2} \phi / \partial y \partial y & \partial^{2} \phi / \partial \theta \partial y \\
\partial^{2} \phi / \partial y \partial \theta & \partial^{2} \phi / \partial \theta \partial \theta
\end{array}\right] .
$$

Proof. By virtue of Lemma 5.5, $d x_{1}, d x_{2}, \ldots d x_{n}$ are linearly independent on $C_{\phi}(U)$. On the other hand on $C_{\phi}(U), d_{(\theta, y)} \phi=0$ and $d\left(\partial \phi / \partial \theta_{1}\right) \ldots, d\left(\partial \phi / \partial \theta_{(N-1) n}\right)$, $d\left(\partial \phi / \partial y_{1}\right) \ldots, d\left(\partial \phi / \partial y_{n}\right)$ are linearly independent in $T^{*} \mathbb{R}^{N(n+1)}$. Therefore $d x_{1}$, $d x_{2}, \ldots, d x_{n}, d\left(\partial \phi / \partial \theta_{1}\right), \ldots, d\left(\partial \phi / \partial y_{n}\right)$ span the whole $T_{(x, \theta, y)}^{*} \mathbb{R}^{n(N+1)}$. This implies obviously that $\operatorname{det} \operatorname{Hess}_{(\theta, y)}(\phi) \neq 0$ on each $(x, \theta, y) \in C_{\phi}(U)$. On the other hand the function $\operatorname{Hess}_{(\theta, y)}(\phi)$ is continuous on $\overline{C_{\phi}(U)}$ and $\overline{C_{\phi}(U)}$ is compact. Combining these facts, we get easily the desired constant $\kappa$ which satisfies (5.27). (Q.E.D.)

Now we can state the main theorem of this section.

Theorem 5.7. Let Assumption $(A)$ be satisfied. Let $f \in \mathscr{H}$ be such that $\operatorname{supp} f \subset U$. Then

$$
\begin{aligned}
& \limsup _{h \downarrow 0} \| e_{t \geq t_{0}}^{-i t H^{h / h}} W_{-}^{h} \tilde{f}_{a}^{h}-e^{-n \pi \nu / 4}|\operatorname{det}(\partial x(t, a, \eta) / \partial \eta)|^{-1 / 2} \\
& \cdot e^{i \operatorname{lnd} t(\gamma(x)) \pi / 2+i S(t, x) / \hbar} f(\eta(t, a, x)) \|=0 .
\end{aligned}
$$

Here $S(t, x)=\int_{-R}^{t} L\left(x_{-}(u, a, \eta(t, a, x)), \dot{x}_{-}(u, a, \eta(t, a, x))\right) d u+S_{-\infty}^{R}(x(-R, a, \eta(t, a, x)))$ and $\operatorname{Ind}_{t} \gamma(x)$ is the so-called Keller-Maslov index of the path $\gamma_{t}=\left\{\left(x_{-}(u, a, x)\right.\right.$, $\left.\left.\xi_{-}(u, a, \eta(t, a, x))\right):-\infty<u \leqq t\right\}$ and is defined explicitly in the proof.

For proving the theorem we need the following lemmas:

Lemma 5.8. Let $a(t, x, y)$ be the function defined in Theorem 5.1. Fix $y \in \mathbb{R}^{n}$ and consider $a(t, x, y)=a(t, x(\eta, y), y)$ as a function of $t$ and $\eta=-\frac{\partial S}{\partial y}(t, x, y)$. Then $a(t, x(t, \eta, y), y)^{2} d x(t, \eta, y)=e^{-i n \pi / 2} d \eta$ for any $0<t<\delta$.

Proof. We first prove that $a(t, x(t, \eta), y)^{2} d x(t, \eta)$ is $t$-independent $n$-form. To see this we differentiate this $n$-form by $t$ and get

$$
\begin{aligned}
\frac{d}{d t} a(t, x(t, \eta, y), y)^{2} d x(t, \eta)= & 2\left((\partial a / \partial t)+\sum_{j=1}^{n}\left(\partial a / \partial x_{j}\right)\left(d x_{j} / d t\right)\right) a(t, x, y) d x \\
& +a(t, x, y)^{2} \sum_{j=1}^{n} d x_{1} \wedge \ldots \wedge \frac{d}{d t}\left(d x_{j}\right) \wedge \ldots \wedge d x_{n} .
\end{aligned}
$$

Since $d / d t\left(d x_{j}\right)=d\left(d x_{j} / d t\right)=d\left(\partial S / \partial x_{j}(t, x, y)\right)=\sum_{k=1}^{n}\left(\partial^{2} S / \partial x_{j} \partial x_{k}\right) d x_{k}$, the right hand side is equal to $a(t, x, y) d x$ multiplied by

$$
2\left(\partial a / \partial t+\sum_{j=1}^{n}\left(\partial a / \partial x_{j}\right)\left(\partial S / \partial x_{j}\right)+2^{-1}(\Delta S) \cdot a\right)
$$

which vanishes identically by the definition of $a(t, x, y)$. Since $\lim _{t \rightarrow 0} t^{-n} d x(t, \eta)=$ $\lim d((x(t, \eta, y)-y) / t)=d \eta$ and the construction of $a(t, x, y)$ shows that $\lim _{t \rightarrow 0} t^{n / 2} a(t, x, y)=e^{-i n \pi / 4}$, we get the desired result.

(Q.E.D.) 
Lemma 5.9. For $(x, \theta, y) \in C_{\phi}(U)$,

$\operatorname{det} \operatorname{Hess}_{(\theta, y)}(\phi)(x, \theta, y)$

$$
\begin{aligned}
& =\operatorname{det} \operatorname{Hess}_{(\theta, y)}\left(\sum_{j=1}^{N} S\left(\tau, x_{j}, x_{j-1}\right)+S_{-\infty}^{R}(y)\right) \\
& =\operatorname{det}(\partial x / \partial y) \cdot \operatorname{det}\left(\partial^{2} S / \partial x_{N} \partial x_{N-1}\right) \ldots \operatorname{det}\left(\partial^{2} S / \partial x \partial_{2} x_{1}\right) \cdot \operatorname{det}\left(\partial^{2} S / \partial x_{1} \partial y\right) .
\end{aligned}
$$

Proof. Since $d_{(\theta, y)} \phi(x(y), \theta(y), y)=0, y \in \Omega_{1,-}^{R, a}(U)$, we have for $j=0, \ldots, N-1$,

$$
\begin{aligned}
(\partial / \partial y)\left(\partial \phi / \partial x_{j}(x(y), \theta(y), y)\right) & =\sum_{k=0}^{N} \partial^{2} \phi / \partial x_{j} \partial x_{k} \cdot \partial x_{k} / \partial y \\
& =0, \text { where } x_{N}=x, x_{0}=y .
\end{aligned}
$$

Hence for $1 \leqq j$,

$$
\begin{aligned}
& \left(\partial^{2} S / \partial x_{j} \partial x_{j+1}\right)\left(\tau, x_{j+1}(y), x_{j}(y)\right) \cdot \partial x_{j+1} / \partial y \\
& \quad+\left(\partial^{2} S / \partial x_{j}^{2}\right)\left(\tau, x_{j+1}(y), x_{j}(y)\right) \cdot \partial x_{j} / \partial y \\
& \quad+\left(\partial^{2} S / \partial x_{j}^{2}\right)\left(\tau, x_{j}(y), x_{j-1}(y)\right) \cdot \partial x_{j} / \partial y \\
& \quad+\left(\partial^{2} S / \partial x_{j} \partial x_{j-1}\right)\left(\tau, x_{j}(y), x_{j-1}(y)\right) \cdot \partial x_{j-1} / \partial y=0
\end{aligned}
$$

and

$$
\begin{aligned}
\partial^{2} S / \partial x_{1} \partial y\left(\tau, x_{1}(y), y\right) \cdot \partial x_{1} / \partial y & +\partial^{2} S / \partial y^{2}\left(\tau, x_{1}(y), y\right) \\
& +\partial^{2} S_{-\infty}^{R} / \partial y^{2}\left(\tau, x_{1}(y), y\right)=0 .
\end{aligned}
$$

By definition

$$
\begin{aligned}
& \operatorname{det} \operatorname{Hess}_{(\theta, v)}(\phi)=
\end{aligned}
$$

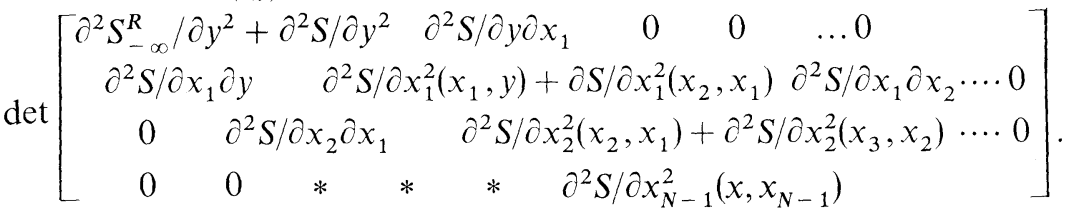

Multiplying the $(j+1)$-th column by $\partial x_{j} / \partial y$ and adding those columns to the first we get by (5.29) and (5.30) that the right hand side of (5.31) is equal to the determinant of the following matrix:

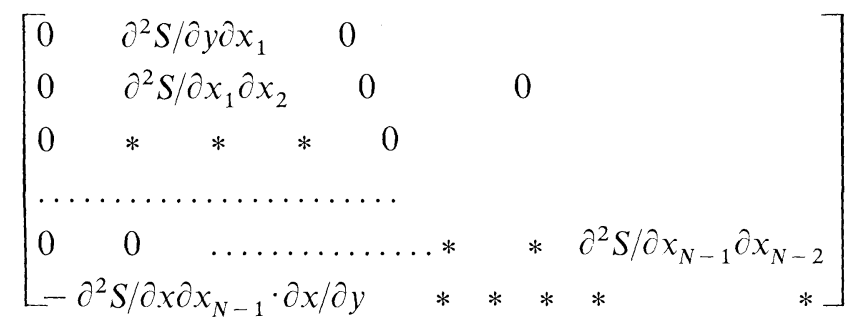

which is equal to the desired quantity.

(Q.E.D.) 


\section{Corollary 5.10}

$$
|\operatorname{det} \operatorname{Hess}(\phi)|^{-1} \prod_{j=1}^{N}\left|a\left(\tau, x_{j}(y), x_{j-1}(y)\right)\right|^{2}=|\operatorname{det}(\partial y / \partial x(y))| .
$$

Proof. Since $\partial^{2} S / \partial x_{j+1} \partial x_{j}=-\partial \xi_{j}(y) / \partial x_{j+1},(5.32)$ is the immediate consequence of Lemma 5.8 and Lemma 5.9.

(Q.E.D.)

Proof of Theorem 5.7. We first prove

$$
\begin{gathered}
\lim _{h \downarrow 0} \| \exp \left(-i t_{0} H^{h} / \hbar\right) W_{-}^{h} \tilde{f}_{a}^{h}-e^{i \operatorname{lnd}(\gamma) \pi / 2+i S\left(t_{0}, x\right) / \hbar-i n \pi / 4} \\
\cdot\left|\operatorname{det}\left(\partial x\left(t_{0}, a, \eta\right) / \partial \eta\right)\right|^{-1 / 2} f\left(\eta\left(t_{0}, a, x\right)\right) \|=0 .
\end{gathered}
$$

By virtue of (5.19), it suffices to prove (5.33) replacing $\exp \left(-i t H^{h} / \hbar\right) W_{-}^{h} \tilde{f}_{a}^{h}$ by $E(\tau, h)^{N} W_{-, a}^{R} f$. Let $\chi(x, \theta, y)$ be an infinitely differentiable function on $\mathbb{R}^{n} \times \mathbb{R}^{(N-1) n} \times \mathbb{R}^{n}$ with compact support such that $\chi(x, \theta, y) \equiv 1$ on a small neighbourhood of $C_{\phi}(U)$. Using the function $\chi(x, \theta, y), E(\tau, h)^{N} W_{-, a}^{R} f$ is divided into two parts:

$$
\begin{aligned}
E(\tau, h)^{N} W_{-a}^{R} f \\
=I_{1}(h) f+I_{2}(h) f \\
=h^{-N n / 2} \int e^{i \hbar^{-1} \phi(x, \theta, y)} A(x, \theta, y) \chi(x, \theta, y) h_{R}(y) d y d \theta \\
\quad+h^{-N n / 2} \int e^{i \hbar^{-1} \phi(x, \theta, y)} A(x, \theta, y)(1-\chi(x, \theta, y)) h_{R}(y) d y d \theta,
\end{aligned}
$$

where $A(x, \theta, y)=\prod_{j=1}^{N} a\left(\tau, x_{j}, x_{j-1}\right)$. We first treat $I_{2}(h) f$. Since $d_{(\theta, y)} \phi \neq 0$ on the support of $1-\chi(x, \theta, y)$, the first order differential operator

$$
L=\left(|\partial \phi / \partial \theta|^{2}+|\partial \phi / \partial y|^{2}\right)^{-1}(\partial \phi / \partial \theta \cdot \partial / \partial \theta+\partial \phi / \partial y \cdot \partial / \partial y)
$$

has a meaning on the support of $1-\chi(x, \theta, y)$, and

$-i \hbar \cdot L\left(e^{i \phi(x, \theta, y) / \hbar}\right)=e^{i \phi(x, \theta, y) / \hbar}$

Therefore

$$
\begin{aligned}
& \int e^{i \hbar^{-1} \phi(x, \theta, y)} A(x, \theta, y)\left(1-\chi(x, \theta, y) h_{R}(y) d y d \theta\right. \\
& \quad=-\int i \hbar \cdot L\left(e^{i \hbar^{-1} \phi(x, \theta, y)}\right) A(x, \theta, y)\left(1-\chi(x, \theta, y) h_{R}(y) d y d \theta\right. \\
& \quad=-\hbar \int i e^{i h^{-1} \phi(x, \theta, y)} L^{*}\left(A(x, \theta, y)(1-\chi(x, \theta, y)) h_{R}(y)\right) d y d \theta
\end{aligned}
$$

Since $h_{R}(y)$ has a compact support and $\phi(t, x, y)$ satisfies (5.3) we easily see that

$$
\begin{aligned}
& L^{*}\left(A(x, \theta, y)(1-\chi(x, \theta, y)) h_{R}(y)\right) \\
& \quad=B_{1}(x, \theta, y) h_{R}(y)+B_{2}(x, \theta, y) \cdot \partial h_{R} / \partial y(y),
\end{aligned}
$$

where $B_{1}(x, \theta, y)$ and $B_{2}(x, \theta, y)$ are the functions in the space $\mathscr{B}\left(\mathbb{R}^{n} \times \mathbb{R}^{n(N-1)} \times \mathbb{R}^{n}\right)$. Therefore by using Theorem 5.1 we get the relation

$$
\left\|I_{2}(h) f\right\| \leqq C h\|f\|_{1}
$$


with suitable constant $C$. (For the rigorous justification of the step from the 2-nd expression to the third in (5.35), the partial integration, we refer to Asada-Fujiwara [2]. Such a justification will be also necessary in calculations in Sect. 6, though we shall not mention it there.) For $I_{1}(h) f$ we use the stationary phase method. First of all we remark that supp $I_{1}(h) f$ is contained in the fixed compact set $\left\{x \in \mathbb{R}^{n}:(x, \theta, y) \in \operatorname{supp} \chi\right.$ for some $\left.(\theta, y) \in \mathbb{R}^{(n-1) N} \times \mathbb{R}^{n}\right\}$. There exists a constant $\kappa>0$ such that $\left|\operatorname{det} \operatorname{Hess}_{(\theta, y)}(\phi)(x, \theta, y)\right| \geqq \kappa$ on $C_{\phi}(U)$. Then the stationary phase method implies

$$
\begin{aligned}
& \mid I_{1}(h) f(x)-e^{\operatorname{Inert}(\operatorname{Hess}(\phi)) \pi i / 2-i n \pi / 4} \cdot e^{i N n \pi / 4} \prod_{j=1}^{N} a\left(\tau, x_{j}(x), x_{j-1}(x)\right) e^{i \phi(x, \theta(x), y(x)) / \hbar} \\
& \left|\operatorname{det} \operatorname{Hess}_{(\theta, y)} \phi(x, \theta(x), y(x))\right|^{-1 / 2} h_{R}(y(x)) \mid \leqq C_{f} h,
\end{aligned}
$$

where Inert $(\operatorname{Hess}(\phi))$ is the inertia of the Hessian of $\phi$ and $C_{f}$ is the constant determined by a suitable Sobolev norm of $h_{R}$, hence by the one of $f$. As in the proof of Lemma 5.3, $x_{j}(x)=x(-R+j \tau, a, \eta(t, a, x)), j=0,1, \ldots, N-1$. Corollary 5.10 shows $\prod_{j=1}^{N} a\left(\tau, x_{j}(x), x_{j-1}(x)\right) \cdot \mid \operatorname{det}\left(\left.\operatorname{Hess}_{(\theta, y)} \phi(x, \theta(x), y(x))\right|^{-1 / 2}=\right.$ $e^{-i N n \pi / 4}|\operatorname{det}(\partial y(x) / \partial x)|^{-1 / 2}$. By the construction of $S(\tau, x, y)$, it is obvious that $\phi(x, \theta(x), y(x))=\sum S\left(\tau, x_{j}(x), x_{j-1}(x)\right)+S_{-\alpha}^{R}(y(x))=S\left(t_{0}, x\right)$. Therefore writing as $\operatorname{Ind}_{t}(\gamma)=\operatorname{Inert}\left(\right.$ Hess $\phi(x, \theta(x), y(x))$, we get for $f \in C_{0}^{\infty}(U)$

$$
\lim _{h \downarrow 0}\left\|I_{1}(h) f-e^{i \operatorname{lnd}(\gamma) \pi / 2+i S(t, x) / \hbar-i n \pi / 4}|\operatorname{det} \partial y(x) / \partial x|^{1 / 2} h_{R}(y(x))\right\|=0 .
$$

(5.37) obviously implies (5.33) for $f \in C_{0}^{\infty}(U)$. Since the relevant operators in (5.33) are isometries, we can get (5.33) for general $f \in \mathscr{H}$ with supp $f \subset U$ by the standard argument.

Let us now prove the relation (5.28). Let $K_{t_{0}}(t, x)=K(t, x)$ be the function defined by (3.13). Then the definition of $K(t, x)$ implies that we can write as

$$
\begin{aligned}
Z_{a}^{h}(t) f(x) \equiv & |\operatorname{det}(\partial x(t, a, \eta) / \partial \eta)|^{-1 / 2} e^{-i n \pi / 4} e^{i \operatorname{Ind}_{t}(\gamma) \pi / 2+i S(t, x) / \hbar} f(\eta(t, a, x)) \\
= & e^{i S(t, x) / \hbar} e^{K(t, x)}\left\{\left|\operatorname{det}\left(\partial x\left(t_{0}, a, \eta\right) / \partial \eta\right)\right|^{-1 / 2} e^{-i n \pi / 4}\right. \\
& \left.\cdot e^{i \operatorname{Ind}(\gamma) \pi / 2} f\left(\eta\left(t_{0}, a, x\left(t_{0}, a, \eta(t, a, x)\right)\right)\right)\right\} \\
\equiv & e^{i S(t, x) /} e^{K(t, x)} g(y(t, x))
\end{aligned}
$$

where $y=x\left(t_{0}, a, \eta(t, a, x)\right)$. Then the similar argument used in the proof of Theorem 4.1 shows that

$$
\begin{aligned}
\left\|e^{-i t H^{h} / h} W_{-}^{h} \tilde{f}_{a}^{h}-Z_{a}^{h}(t) f\right\| \leqq & C h \int_{t_{0}}^{t}\left\|\Delta_{x}\left(e^{K(u, x)} g(y(u, x))\right)\right\| d u \\
& +\left\|e^{-i t_{0} H^{h} / h} W_{-}^{h} \tilde{f}_{a}^{h}-Z_{a}^{h}\left(t_{0}\right) f\right\| .
\end{aligned}
$$

By (3.14) through (3.17) and the similar formula as (4.6), the first summand of the right hand side of (5.39) can be estimated as follows:

$$
\begin{aligned}
C h \int_{t_{0}}^{t} \| \Delta_{x}\left(e^{K(u, x)} g(y(u, x)) \| d u\right. & \leqq C h \int_{t_{0}}^{t} u^{-2} \log (u)\|g\|_{2} d u \\
& \leqq C M h\|f\|_{2},
\end{aligned}
$$


where $C$ and $M$ are the constants depending only on $t_{0}$. From (5.39), (5.40) and the result of the first part of the proof of this theorem, we can get the statement of the theorem by the standard argument.

(Q.E.D.)

\section{The Quasi-Classical Limit of the Scattering Operator}

Using the materials studied in the previous sections, we study here the asymptotic behavior of the scattering operator $S^{h}=\left(W_{+}^{h}\right)^{*} W_{-}^{h}$ on the coherent state $\tilde{f}_{a}^{h}$. We study it in the momentum space representation, that is, we study the asymptotic behavior of

$$
\hat{S}^{h}\left(e^{-i p \cdot a / \hbar} f(p)\right)=\mathscr{F}^{h} S^{h} \mathscr{F}^{h^{*}}\left(e^{-i p \cdot a / \hbar} f(p)\right) .
$$

Under the Assumption (A), $\eta_{+}=\eta_{+}(\eta)$ is a smooth function of $\eta \in \mathbb{R}^{n} \backslash e(a)$. We define as

$$
e(a)^{e x}=\left\{\eta \in \mathbb{R}^{n} \backslash e(a): \operatorname{det} \partial \eta_{+}(\eta) / \partial \eta=0\right\} \cup e(a)
$$

Obviously the set $e(a)^{e x}$ is a closed subset of $\mathbb{R}^{n}$.

Theorem 6.1. Let Assumption (A) be satisfied. Let $K \subset \subset \mathbb{R}^{n} \backslash e(a)^{e x}$. Then the following statements hold.

1) For any $\eta_{+} \in S_{a}^{c l}(K)$, there exists at most finite number of $\eta_{j}=\eta_{j}\left(\eta_{+}\right) \in K$ such that $\eta_{+}=S_{a}^{c l}\left(\eta_{j}\right)$.

2) Let $f \in \mathscr{H}$ be such that $\operatorname{supp} f \subset K$. Then

$$
\begin{aligned}
\lim _{h \downarrow 0} \| & \hat{S}^{h} f_{a}^{h}\left(\eta_{+}\right)-\left.\sum_{j} e^{i \operatorname{Ind} \gamma\left(\eta_{+}, \eta_{J}\right) \pi / 2}\left|\operatorname{det} \partial \eta_{+}(\eta) / \partial \eta\right|^{-1 / 2}\right|_{\eta=\eta_{J}(\eta+)} \\
\cdot & e^{i\left(S\left(\eta_{+}, \eta_{J}\right)-\eta_{+} \cdot a_{+}\left(\eta_{J}\left(\eta_{+}\right)\right)\right) / \hbar} f\left(\eta_{j}\left(\eta_{+}\right)\right) \|=0,
\end{aligned}
$$

where Ind $\gamma\left(\eta_{+}, \eta_{j}\right)$ is the Keller-Maslov's index of the orbit $\left(x_{-}\left(t, a, \eta_{j}\right), \xi_{-}\left(t, a, \eta_{j}\right)\right.$ : $-\infty<t<\infty)$,

$$
S\left(\eta_{+}, \eta_{j}\right)=\lim _{\substack{t \rightarrow \infty \\ s \rightarrow-\infty}}\left\{\int_{s}^{t} L\left(x_{-}\left(u, a, \eta_{j}\right), \dot{x}_{-}\left(u, a, \eta_{j}\right)\right) d u+s \eta_{j}^{2} / 2 m-t \eta_{+}^{2} / 2 m\right\} .
$$

Proof. We assume $m=1$. Let us take $K_{1}$ as $K \subset \subset K_{1} \subset \subset \mathbb{R}^{n} \backslash e(a)^{e x}$. Since $K_{1}$ is precompact there exists a constant $\delta_{K_{1}}>0$ such that (3.11) holds for every $\eta \in K_{1}$. Then by the remark following (3.11), the first statement of the theorem is obvious. As in that remark, let us take finite precompact subsets $\left\{U_{j}\right\}$ such that $\left\{U_{j}\right\}$ is a covering of $K$ and $S_{a}^{c l}$ is a diffeomorphism on each $U_{j}$. It is sufficient to prove the relation (6.2) for $f \in \mathscr{H}$ with supp $f \subset U_{j}$, for some $U_{j}$. Moreover, since in this case the operators appearing in (6.2) are isometric, we may assume that $f \in C_{0}^{\infty}(U)$. By virtue of Theorem 5.7 and by the unitarity of the propagator $\exp \left(i H_{0}^{h} / \hbar\right)$ and the Fourier transform $\mathscr{F}^{h}$, we get

$$
\lim _{h \downarrow 0} \sup _{t \geqq t_{0}}\left\|\mathscr{F}^{h} e^{i t H_{O}^{h} / \hbar} e^{-i t H^{h} / h} W_{-}^{h} \tilde{f}_{a}^{h}-\mathscr{F}^{h} e^{i t H_{O}^{h / \hbar}} Z_{a}^{h}(t) f\right\|=0 .
$$

Here we used the notation in the proof of Theorem 5.7 for $Z_{a}^{h}(t)$. The second term 
of the left hand side of (6.4) can be written as

$$
\mathscr{F}^{h} e^{i t H_{0}^{h} / \hbar} Z_{a}^{h}(t) f=e^{i t p^{2} / 2 \hbar} \mathscr{F}^{h} Z_{a}^{h}(t) f .
$$

To the right hand side of (6.5) we apply the stationary phase method. Using the notation of Theorem 5.7 and its proof, let us write as

$$
\begin{aligned}
h(t, x) & =e^{i \operatorname{lnd} d_{t} \gamma(x) \pi / 2}|\operatorname{det}(\partial x(t, a, \eta) / \partial \eta)|^{-1 / 2} e^{-i n \pi / 4} f(\eta(t, a, x)) \\
& =e^{K(t, x)} g(y(x, t)) .
\end{aligned}
$$

Then

$$
\mathscr{F}^{h} Z_{a}^{h}(t) f(\eta)=h^{-n / 2} \int e^{i \hbar^{-1}(S(t, x)-x \cdot \eta)} h(t, x) d x .
$$

Making the change of variable $x$ to $x t$ and writing $v=\hbar t^{-1}, \phi(t, x, \eta)=$ $\left(S(t, t x) t^{-1}-x \cdot \eta\right)$, and $h_{t}(x)=t^{n / 2} h(t, t x)$, we have

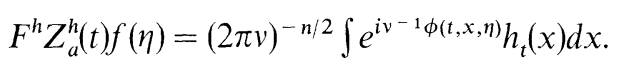

For each $\eta \in \mathbb{R}^{n}$, the point of stationary phase is determined by the equation $d_{x} \phi(t, x, \eta)=0$, that is,

$$
\partial S / \partial x(t, t x)=\eta
$$

Since $\partial S / \partial x(t, t x)=\xi_{-}(t, a, \eta(t, a, t x))$ and the mappings $\eta \rightarrow \xi_{-}(t, a, \eta)$ and $\eta \rightarrow x_{-}(t, a, \eta)$ are both diffeomorphisms on $U$ by Lemma 2.7 and Lemma 3.3, we can easily see that if $\eta \in \pi_{2} U(t+R) W_{-, R}^{c l}(U)$, there exists a unique point of stationary phase $x=x_{t}(\eta) \equiv x_{-}(t, a, \eta) / t$ and otherwise there is no point of stationary phase. Let us write as

$$
C_{t}(U)=\left\{\left(x_{t}(\eta), \eta\right): \eta \in \pi_{2} U(t+R) W_{-, R}^{c l}(U)\right\}
$$

By Lemma 2.7 and Lemma 3.3, we can see that there is a precompact subset $V \subset \mathbb{R}^{n} \times \mathbb{R}^{n}, W \subset K \subset W_{1} \subset K_{1}$ such that $\bar{V}$ is a neighbourhood of the diagonal set of $S_{a}^{c l}(W) \times S_{a}^{c l}(W)$ contained in $S_{a}^{c l}\left(W_{1}\right) \times S_{a}^{c l}\left(W_{1}\right)$ such that $\bigcup_{t \geqq t_{0}} C_{t}(U) \subset \subset V$.

Let us take as infinitely differentiable function $\chi(x, \eta)$ on $\mathbb{R}^{n} \times \mathbb{R}^{n}$ with compact support such that $\chi(x, \eta) \equiv 1$ on $V$. Using the function $\chi(x, \eta)$ we divide $\mathscr{F}^{h} Z_{a}^{h}(t) f(\eta)$ into two parts:

$$
\begin{aligned}
\mathscr{F}^{h} Z_{a}^{h}(t) f(\eta)= & (2 \pi v)^{-n / 2} \int e^{i v-1 \phi(t, x, \eta)} \chi(x, \eta) h_{t}(x) d x \\
& +(2 \pi v)^{-n / 2} \int e^{i v^{-1} \phi(t, x, \eta)}(1-\chi(x, \eta)) h_{t}(x) d x \\
= & I_{1}(t, v) f(\eta)+I_{2}(t, v) f(\eta) .
\end{aligned}
$$

We should remark here that in each integral of (6.10), there exists a compact subset of $\mathbb{R}_{x}^{n}$ such that the support of function $h_{t}(x)$ is contained in it for $t \geqq t_{0}$ by Lemma 2.7 . We first study $I_{2}(t, v) f(\eta)$. Define the first order differential operator $L_{t}$ by

$$
L_{t}=\left(\sum_{j=1}^{n}\left(\partial \phi / \partial x_{j}\right)^{2}\right)^{-1} \sum_{j=1}^{n}\left(\partial \phi / \partial x_{j}\right) \cdot \partial / \partial x_{j}
$$


which satisfies

$$
-i v L_{t}\left(e^{i v^{-1} \phi(t, x, \eta)}\right)=e^{i v^{-1} \phi(t, x, \eta)}
$$

and

$$
\begin{aligned}
L_{t}^{*}= & -L_{t}-\left\{\left(\sum_{j=1}^{n}\left(\partial \phi / \partial x_{j}\right)^{2}\right)^{-1} \Delta \phi\right. \\
& \left.-2\left(\sum_{j=1}^{n}\left(\partial \phi / \partial x_{j}\right)^{2}\right)^{-2} \sum_{j, k=1}^{n}\left(\partial \phi / \partial x_{j}\right)\left(\partial \phi / \partial x_{k}\right)\left(\partial^{2} \phi / \partial x_{k} \partial x_{j}\right)\right\}
\end{aligned}
$$

Hence writing the second term of the r.h.s of (6.12) as $G(t, x, p)$,

$$
\begin{aligned}
& (2 \pi)^{n / 2} I_{2}(t, v) f(\eta)=-i v \cdot v^{-n / 2} \int L_{t}\left(r^{i v-1} \phi(t, x, \eta)\right)(1-\chi(x, \eta)) h_{t}(x) d x \\
& =-i v \cdot v^{-n / 2} \int e^{i v^{-1} \phi(t, x, \eta)} L_{t}^{*}\left((1-\chi(x, \eta)) h_{t}(x)\right) d x \\
& =i v \cdot v^{-n / 2} \int e^{i v-1} \phi(t, x, \eta)(1-\chi(x, \eta)) L_{t}\left(h_{t}(x)\right) d x \\
& +i v \cdot v^{-n / 2} \int e^{i v-1} \phi(t, x, \eta)\left\{L_{t}(1-\chi(x, \eta))+G(t, x, \eta)\right\} h_{t}(x) d x \\
& =I I_{1}+I I_{2} \text {. }
\end{aligned}
$$

By Lemma 2.7 and Lemma 3.3 we can easily see that

$$
L_{t}(1-\chi(x, \eta))+G(t, x, \eta) \in \mathscr{B}\left(\mathbb{R}^{n} \times \mathbb{R}^{n}\right),
$$

uniformly in $t \geqq t_{0}$, and $\phi(t, x, \eta)$ obviously satisfies (5.13) uniformly in $t \geqq t_{0}$ (taking the number of variable $\theta=m=0$ ). Hence by Theorem 5.2, we get

$$
\left\|I_{2}\right\| \leqq C v\left\|h_{t}(x)\right\| \leqq C v\|h(t, x)\| \leqq C v\|f\| .
$$

To estimate $I I_{1}$, we first note that, using the terminology (5.38),

$$
\begin{aligned}
\partial / \partial x_{j}\left(h_{t}(x)\right)= & \partial / \partial x_{j}\left(e^{K(t, t x)} g(y(t, t x))\right) t^{n / 2} \\
= & t\left(\partial K / \partial x_{j}\right) e^{K(t, t x)} g(y(t, t x)) t^{n / 2} \\
& +e^{K(t, t x)}(\partial g / \partial y)(y(t, t x)) \cdot t(\partial y / \partial x) t^{n / 2} .
\end{aligned}
$$

Since $(1-\chi(x, \eta))\left(\sum\left(\partial \phi / \partial x_{j}\right)^{2}\right)^{-1} \partial \phi / \partial x_{j} \in \mathscr{B}\left(\mathbb{R}^{n} \times \mathbb{R}^{n}\right)$ uniformly in $t ;\left|\partial K / \partial x_{j}\right|=$ $t\left|\sum\left(\partial K / \partial y_{k}\right) \cdot\left(\partial y_{k} / \partial x_{j}\right)\right| \leqq C t \cdot t^{-1} \cdot \log t=C \log t$ and $t|\partial y / \partial x| \leqq C$ by Lemma 2.7 and Lemma 3.3, we get again by Theorem 5.2 that

$$
\begin{aligned}
\left\|I I_{1}\right\| & \leqq C v\left(\log t\left\|h_{t}(x)\right\|+\left\|e^{K(t, x)}(\partial g / \partial y)(y(t, x))\right\|\right) \\
& \leqq C h / t\left(\log t\|f\|+\|f\|_{1}\right)
\end{aligned}
$$

Summing up the relations (6.14) and (6.15), we get

$$
\left\|I_{2}(t, y) f\right\| \leqq C h t^{-1} \log t\|f\|_{1} \text {. }
$$

Now we turn to the study of $I_{1}(t, v) f(\eta)$. Since $C_{t}(U)$ converges to $C_{\infty}(U)$, we may assume that for any $t \geqq t_{0}$ and $\eta \in \pi_{2} V$, there exists $x_{t}(\eta) \in S_{a}^{c l}\left(W_{1}\right)$ such that $0=d_{x} \phi\left(t, x_{t}(\eta), \eta\right)$. For $\left(x_{t}(\eta), \eta\right)$ the function $\phi(t, x, \eta)$ can be written as

$$
\phi(t, x, \eta)=\phi\left(t, x_{t}(\eta), \eta\right)+\left(x-x_{t}(\eta)\right) \cdot B(t, x, \eta)\left(x-x_{t}(\eta)\right) .
$$


where $B(t, x, \eta)=\int_{0}^{1}(1-u) \partial^{2} \phi / \partial x^{2}\left(t, u x+x_{t}(\eta)(1-u), \eta\right) d u$. Since Hess $\operatorname{He}_{x} \phi(t, x, \eta)=$ $t\left(\operatorname{Hess}_{x} S\right)(t, t x)=t\left(\partial \xi_{-} / \partial x\right)(t, a, \eta(t, a, t x))$

$$
=\partial \xi_{-}(t, a, \eta) /\left.\partial \eta\right|_{\eta=\eta(t, a, x t)} t\left(\partial x_{-}(t, a, \eta) /\left.\partial \eta\right|_{\eta=\eta(t, a, t x)}\right)^{-1},
$$

Lemma 2.7 and Lemma 3.3 imply that the family $\left\{\operatorname{Hess}_{x} \phi(t, \ldots,)\right\}_{t \geq t_{0}}$ is equicontinuous and are non-singular on $\bar{V}$. Therefore by Morse's lemma, implicit function theorem and a simple compactness argument, it follows that there exists a finite number of subsets $V_{k}$ of $\mathbb{R}^{n}$ such that each $V_{k}$ is precompact open set, $\cup V_{k} \supset \bar{V}$ and on each $V_{k}$ there exists a change of variable $x$ to $y=y(x, t, \eta)$ such that $\{y(x, t, \eta)\}_{t \geqq t_{0}}$ is a bounded set of $\mathscr{B}\left(\mathbb{R}^{n} \times \mathbb{R}^{n}\right)$ with its inverse, $y\left(x_{t}(\eta), t, \eta\right)=0$, det $\partial x / \partial y\left(x_{t}(\eta), t, \eta\right)=1$ and $B(t, x, \eta)=2^{-1} \sum d_{j}(t, \eta) y^{2}$, where $d_{j}(t, \eta)$ 's are the eigenvalues of the matrix $\operatorname{Hess}_{x} \phi\left(t, x_{t}(\eta), \eta\right)$. Let $\left\{\tilde{\omega}_{k}(x, \eta)\right\} \subset$ $C_{0}^{\infty}\left(\mathbb{R}^{n} \times \mathbb{R}^{n}\right)$ be the partition of unity on $V$ subordinate to the covering $\left\{V_{k}\right\}, \omega_{k}(x, \eta)=\tilde{\omega}_{k}(x, \eta) \chi(x, \eta)$. Using the function $\omega_{j}(x, \eta)$ we divide $I_{1}(t, v) f(\eta)$ as

$$
\begin{aligned}
& I_{1}(t, v) f(\eta)=\sum_{k} I_{1, k}(t, v) f(\eta) \\
& =\sum_{k}(2 \pi v)^{-n / 2} \int e^{i \nu-1} \phi(t, x, \eta) \omega_{k}(x, \eta) h(t, t x) t^{n / 2} d x \\
& =\sum_{k}(2 \pi v)^{-n / 2} \int e^{i v-1} \phi\left(t, x_{t}(\eta), \eta\right)+i v-12^{-1} \sum d_{J} y_{J}^{2} \\
& \cdot t^{n / 2} \omega_{k}(x(y, \eta, t), \eta) h(t, t x(y, \eta, t)) \cdot \operatorname{det}(\partial x / \partial y) d y \\
& =\sum_{k} e^{i v^{-1} \phi\left(t, x_{t}(\eta), \eta\right)}(2 v \pi)^{-n / 2} \int e^{i v-12^{-1} \sum d_{J} y_{j}^{2}} h_{k}(t, y, \eta) d y,
\end{aligned}
$$

where $h_{k}(t, y, \eta)=t^{n / 2} \omega_{k}(x(y, \eta, t), \eta) h(t, t x(y, \eta, t)) \operatorname{det}(\partial x / \partial y)$.

Since $\left(\operatorname{Hess}_{x} \phi(t, x, \eta)\right)^{-1}$ is uniformly bounded in $(t, x, \eta) \in\left\{t \geqq t_{0}\right\} \times \bar{V}, y(t, x, \eta)$ forms a bounded set of $\mathscr{B}\left(\mathbb{R}^{n} \times \mathbb{R}^{n}\right)$ for $t \geqq t_{0}$ with its inverse function. Furthermore there exists constants $C_{1}$ and $C_{2}$ such that $C_{1} \leqq\left|d_{j}(t, \eta)\right| \leqq C_{2}$ on the support of $h_{R}(t, y, \eta)$. Therefore by a similar argument used in the proof of Theorem 1.1 we get

$$
\begin{aligned}
& \| I_{1, k}(t, v) f(\eta)-e^{i \nu^{-1} \phi\left(t, x_{t}(\eta), \eta\right)+i n \pi / 4} t^{n / 2}\left|\operatorname{det}\left(\operatorname{Hess}_{x} \phi\left(t, x_{t}(\eta), \eta\right)\right)\right|^{-n / 2} \\
& \cdot \omega_{k}\left(x_{t}(\eta), \eta\right) h\left(t, x_{t}(\eta) t\right) \\
& \leqq C v\left\|\int_{0}^{1} d s \int(v s)^{-n / 2} e^{i(v s)^{-1} \sum d_{j} y_{j}^{2}} \sum_{l} d_{l}(t, \eta)\left(\partial^{2} / \partial y_{l}^{2}\right) h_{k}(t, y, \eta) d y\right\|
\end{aligned}
$$

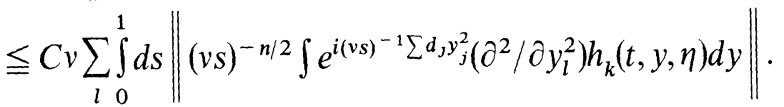

Here we used that $\operatorname{det}\left(\operatorname{Hess}_{x} \phi\left(t, x_{t}(\eta), \eta\right)\right)>0$ which follows from Lemma 2.7.

After changing back variable $y$ to $x$, the integral in the third of (6.19) can be written in the form

$$
\sum_{\mid \alpha\} \leqq 2}(v s)^{-n / 2} \int e^{i(v s)^{-1} \phi(t, x, y)} a_{\alpha}(t, x, \eta)(\partial / \partial x)^{\alpha} h(t, t x) t^{n / 2} d x
$$

where the functions $a_{\alpha}(t, x, \eta)$ are contained in a bounded set of $\mathscr{B}\left(\mathbb{R}^{n} \times \mathbb{R}^{n}\right)$ for 
$t>t_{0}$. Since $(\partial / \partial x)^{\alpha} h(t, t x) t^{n / 2}=t^{n / 2}\left\{(\partial / \partial x)^{\alpha}\left(e^{K(t, t x)} g(y(t, t x))\right)\right\}$ and $\left|(\partial / \partial x)^{\alpha} K(t, t x)\right| \leqq$ $C \log t$, we get $\left\|(\partial / \partial x)^{\alpha} g(y(t, t x)) \cdot \exp (K(t, t x))\right\| \leqq C\|g\|_{2} \leqq C\|f\|_{2}$ and $\left\|(\partial / \partial x)^{\alpha} h(t, x) t^{n / 2}\right\| \leqq C \log t$. Therefore again by Theorem 5.2, we get that the right hand side of (6.19) can be estimated by $C h t^{-1}(\log t)$. Combining these estimates with the estimate for $I_{2}(t, v) f$ we finally get the following estimate:

$$
\begin{aligned}
\lim \sup _{h \downarrow 0} \| & \mathscr{F}_{t \geqq t_{0}}^{h} e^{i t H_{b}^{h} / \hbar} e^{-i t H^{h} / \hbar} W_{-}^{h} \tilde{f}_{a}^{h}\left(\eta_{+}\right) \\
& -e^{i \operatorname{Ind}_{t} \gamma(t, \eta+)} e^{i t \eta^{2} / 2 \hbar} e^{i\left(S\left(t, x_{t}(\eta+) t\right)-t x_{t}(\eta+) \cdot \eta_{+}\right) / \hbar} \\
& \left.\cdot\left|\operatorname{det} \partial \eta_{+}(t, a, \eta) / \partial \eta\right|_{\eta=\eta\left(t, a, \eta_{+}\right)}\right|^{-1 / 2} f\left(\eta\left(t, a, \eta_{+}\right)\right) \|=0 .
\end{aligned}
$$

Since $t x_{t}\left(\eta_{+}\right)=x_{-}\left(t, a, \eta\left(t, a, \eta_{+}\right)\right), t x_{t}\left(\eta_{+}\right) \eta_{+}-t \eta_{+}^{2} / 2-a_{+}\left(a, \eta_{+}\right) \eta_{+}=\left(x_{-}(t, a\right.$, $\left.\left.\eta\left(t, a, \eta_{+}\right)\right)-t \eta_{+}-a_{+}\left(a, \eta_{+}\right)\right) \eta_{+}$converges to zero as $t \rightarrow \infty$, we get the desired relation, taking the limit $t \rightarrow \infty$ in (6.20).

(Q.E.D.)

\section{Concluding Remarks}

Remark 1. We first want to explain here the technical reason why we took $e^{-i \eta \cdot a / \hbar} f(\eta)$ as the initial state instead of $e^{i x \cdot \xi / \hbar} f(x)$ which, in the limit $h \rightarrow 0$, gives the ensemble of the classical particles with the fixed momentum $\xi$ and the configuration distribution density $|f(x)|^{2}$. Indeed the fixed momentum scattering would be more suitable in the classical mechanics because of its popularity. However, in spite of the fact that as $h \rightarrow 0$ the state $e^{i x \cdot \xi / \hbar} f(x)$ is propagated by the free motion $\exp \left(i t H_{0}^{h} / \hbar\right)$ as the ensemble of the classical particles with the fixed momentum $\xi$ in finite time region, it is not uniform in $t>\delta>0$. We can see it from the following remarkable fact: for any $f \in \mathscr{H}$,

$$
\lim _{t \rightarrow \pm \infty}\left\|e^{i t H_{0}} f(x)-(m / i t)^{n / 2} e^{i m x^{2} / 2 t} \hat{f}(m x / t)\right\|=0 .
$$

The relation (7.1) implies that we cannot prepare the ensemble of the fixed momentum incoming classical particles from the quantum mechanics, at least in $L^{2}$-theoretical framework.

Remark 2. In the theorem we have the finite sum over the incoming momentums with the same outgoing one. These summands are asymptotically orthogonal each other as $h \rightarrow 0$. This can be easily seen as follows. Since the classical mechanical scattering operator is a canonical mapping on $\mathbb{R}^{n} \times \mathbb{R}^{n} \backslash e$ we have $a_{+}\left(\eta_{j}\right)=$ $\left(-\frac{\partial}{\partial \eta_{+}}\right)\left(S\left(\eta_{+}, \eta_{j}\right)-a_{+}\left(\eta_{j}\right) \cdot \eta_{+}\right) \neq\left(-\frac{\partial}{\partial \eta_{+}}\right)\left(S\left(\eta_{+}, \eta_{k}\right)-a_{+}\left(\eta_{k}\right) \cdot \eta_{+}\right)=a_{+}\left(\eta_{k}\right) \quad$ if $\eta_{k} \neq \eta_{j}$. Therefore by the stationary phase method we can see that they are asymptotically orthogonal each other as $h \rightarrow 0$. Hence if $f \in \mathscr{H}$ has its support supp $f \subset \subset$ $\mathbb{R}^{n} \backslash e(a)^{e x}$,

$$
\lim _{h \rightarrow 0} \int_{D}\left|S^{h}\left(e^{-i \eta \cdot a / h} f(\cdot)\right)(\eta)\right|^{2} d \eta=\int_{\left(S_{u}^{u}\right)^{-1} D}|f(\eta)|^{2} d \eta
$$

and (7.2) is still true for $f \in \mathscr{H}$ with supp $f \subset \mathbb{R}^{n} \backslash e(a)^{e x}$ (standard argument). Thanks to this relation we may say that $\sum_{j}\left|\partial \eta_{+}\left(\eta_{j}\right) / \partial \eta_{j}\right|^{-1}$ gives the "differential cross section" associated with this classical scattering process. 
Remark 3. If $f \in H^{2}\left(\mathbb{R}^{n}\right)$ and supp $f \subset \subset \mathbb{R}^{n} \backslash e(a)^{e x}$, the theorem can be improved so that the norm before taking limit $h \rightarrow 0$ is estimated by a constant times $h\|f\|_{2}$. This is an obvious consequence of our calculus.

Remark 4. A more general situation where the initial state is $e^{-i S(\eta) / h} f(\eta)$ with general $S \in C^{\infty}\left(\mathbb{R}^{n}\right)$ and $V(x)$ is a long range potential will be discussed in the forthcoming paper [14].

Acknowledgements. Most part of this work was done while the author was on leave from the University of Tokyo and visiting the ETH-Zürich. It is a pleasure to express his sincere thanks to Professors B. Eckmann and W. Hunziker for giving him a chance to work in Zürich and the Department of Mathematics of the University of Tokyo for permitting his visit.

He also thanks Mr. M. Loss for fruitful discussions and the members of the Institute of Theoretical Physics for their interest in the work. Last, but not least, thanks to Prof. S.T. Kuroda for his unceasing encouragement.

\section{References}

1. Agmon, S. : Spectral Properties of Schrödinger operators and scattering theory. Ann. Scuola Nor. Pisa, Ser IV, 2.2, 151-218 (1975)

2. Asada, K., Fujiwara, D. : On some oscillatory integral transformations in $L^{2}\left(\mathbb{R}^{n}\right)$. Jpn. J. Math. 4, 299-361 (1978)

3. Fujiwara, D. : A construction of the fundamental solution for Schrödinger equation. J. d'Analyse Math. (In press)

4. Hepp, K. : The classical limit for quantum mechanical correlation functions. Commun. Math. Phys. 35, 265-277 (1974)

5. Hepp, K. : On the classical limit in Quantum mechanics. Unpublished note, ETH-Zürich (1974)

6. Herbst, W. : Classical scattering with long range forces. Commun. Math. Phys. 35, 193--214 (1974)

7. Hörmander, L. : Fourier integral operators. Acta. Math. 127, 79-183 (1971)

8. Hörmander, L. : The existence of wave operators in scattering theory. Math. Z. 146, 69-91 (1976)

9. Hunziker, W. : The S-matrix in classical mechanics. Commun. Math. Phys., 8, 283-299 (1968)

10. Kuroda, S. T. : Scattering theory for differential operators. J. Math. Soc. Japan 25, 75-104 (1973)

11. Maslov, V.P. : Théorie des perturbations et méthodes asymptotiques (Translation from Russian) Paris: Dunod 1972

12. Simon, B. : Wave operators for classical particle scattering. Commun. Math. Phys. 23, 37 48 (1971)

13. Dollard, J. D. : Scattering into cones. I. Potential scattering. Commun. Math. Phys. 12, 193-203 (1969)

14. Yajima, K. : The quasi-classical limit of quantum scattering theory. II. Long range scattering. Preprint, University of Virginia (1978)

15. Landau, L. D., Lifschitz, E. M. : Course of theoretical physics, Vol. 1, Mechanics (Translation from Russian). New York: Pergamon Press 1969.

Communicated by B. Simon

Received January 25, 1979 
